\title{
Planck early results. XI. Calibration of the local galaxy cluster Sunyaev-Zeldovich scaling relations ${ }^{\star}$
}

Planck Collaboration: P. A. R. Ade ${ }^{73}$, N. Aghanim ${ }^{48}$, M. Arnaud ${ }^{59}$, M. Ashdown ${ }^{57,4}$, J. Aumont ${ }^{48}$, C. Baccigalupi ${ }^{71}$, A. Balbi $^{31}$, A. J. Banday ${ }^{78,7,64}$, R. B. Barreiro ${ }^{54}$, M. Bartelmann ${ }^{77,64}$, J. G. Bartlett ${ }^{3,55}$, E. Battaner ${ }^{79}$, K. Benabed ${ }^{49}$, A. Benoît ${ }^{47}$, J.-P. Bernard ${ }^{78,7}$, M. Bersanelli ${ }^{28,42}$, R. Bhatia ${ }^{5}$, J. J. Bock ${ }^{55,8}$, A. Bonaldi ${ }^{38}$, J. R. Bond ${ }^{6}$, J. Borrill ${ }^{63,75}$, F. R. Bouchet ${ }^{49}$, H. Bourdin ${ }^{31}$, M. L. Brown ${ }^{4,57}$, M. Bucher ${ }^{3}$, C. Burigana ${ }^{41}$, P. Cabella ${ }^{31}$, J.-F. Cardoso ${ }^{60,3,49}$, A. Catalano ${ }^{3,58}$, L. Cayón ${ }^{21}$, A. Challinor ${ }^{51,57,11}$, A. Chamballu ${ }^{45}$, L.-Y Chiang ${ }^{50}$, C. Chiang ${ }^{20}$, G. Chon ${ }^{65,4}$, P. R. Christensen ${ }^{68,32}$, E. Churazov ${ }^{64,74}$, D. L. Clements ${ }^{45}$, S. Colafrancesco ${ }^{39}$, S. Colombi ${ }^{49}$, F. Couchot ${ }^{62}$, A. Coulais ${ }^{58}$, B. P. Crill ${ }^{55,69}$, F. Cuttaia ${ }^{41}$, A. Da Silva ${ }^{10}$, H. Dahle ${ }^{52,9}$, L. Danese ${ }^{71}$, P. de Bernardis ${ }^{27}$, G. de Gasperis ${ }^{31}$, A. de Rosa ${ }^{41}$, G. de Zotti ${ }^{38,71}$, J. Delabrouille ${ }^{3}$, J.-M. Delouis ${ }^{49}$, F.-X. Désert ${ }^{44}$, J. M. Diego ${ }^{54}$, K. Dolag ${ }^{64}$, S. Donzelli ${ }^{42,52}$, O. Doré ${ }^{55,8}$, U. Dörl ${ }^{64}$, M. Douspis ${ }^{48}$, X. Dupac $^{35}$, G. Efstathiou ${ }^{51}$, T. A. Enßlin ${ }^{64}$, F. Finelli4 ${ }^{41}$, I. Flores-Cacho ${ }^{53,33}$, O. Forni ${ }^{78,7}$, M. Frailis ${ }^{40}$, E. Franceschi ${ }^{41}$, S. Fromenteau ${ }^{3,48}$, S. Galeotta ${ }^{40}$, K. Ganga ${ }^{3,46}$, R. T. Génova-Santos ${ }^{53,33}$, M. Giard ${ }^{78,7}$, G. Giardino ${ }^{36}$, Y. Giraud-Héraud ${ }^{3}$, J. González-Nuevo ${ }^{71}$, K. M. Górski ${ }^{55,81}$, S. Gratton ${ }^{57,51}$, A. Gregorio ${ }^{29}$, A. Gruppuso ${ }^{41}$, D. Harrison ${ }^{51,57}$, S. Henrot-Versillé ${ }^{62}$, C. Hernández-Monteagudo ${ }^{64}$, D. Herranz ${ }^{54}$, S. R. Hildebrandt ${ }^{8,61,53}$, E. Hivon ${ }^{49}$, M. Hobson ${ }^{4}$, W. A. Holmes ${ }^{55}$, W. Hovest ${ }^{64}$, R. J. Hoyland ${ }^{53}$, K. M. Huffenberger ${ }^{80}$, A. H. Jaffe ${ }^{45}$, W. C. Jones ${ }^{20}$, M. Juvela ${ }^{19}$, E. Keihänen ${ }^{19}$, R. Keskitalo ${ }^{55,19}$, T. S. Kisner ${ }^{63}$, R. Kneiss ${ }^{34,5}$, L. Knox ${ }^{23}$, H. Kurki-Suonio ${ }^{19,37}$, G. Lagache ${ }^{48}$, J.-M. Lamarre ${ }^{58}$, J. Lanoux ${ }^{78,7}$, A. Lasenby ${ }^{4,57}$, R. J. Laureijs ${ }^{36}$, C. R. Lawrence ${ }^{55}$, S. Leach ${ }^{71}$, R. Leonardi ${ }^{35,36,24}$ A. Liddle $^{18}$, P. B. Lilje ${ }^{52,9}$, M. Linden-Vørnle ${ }^{13}$,

M. López-Caniego ${ }^{54}$, P. M. Lubin ${ }^{24}$, J. F. Macías-Pérez ${ }^{61}$, C. J. MacTavish ${ }^{57}$, B. Maffei ${ }^{56}$, D. Maino ${ }^{28,42}$, N. Mandolesi ${ }^{41}$, R. Mann ${ }^{72}$, M. Maris ${ }^{40}$, F. Marleau ${ }^{15}$, E. Martínez-González ${ }^{54}$, S. Masi ${ }^{27}$, S. Matarrese ${ }^{26}$, F. Matthai ${ }^{64}$, P. Mazzotta ${ }^{31}$, A. Melchiorri ${ }^{27}$, J.-B. Melin ${ }^{12}$, L. Mendes $^{35}$, A. Mennella ${ }^{28,40}$, S. Mitra ${ }^{55}$, M.-A. Miville-Deschênes ${ }^{48,6}$, A. Moneti ${ }^{49}$, L. Montier ${ }^{78,7}$, G. Morgante ${ }^{41}$, D. Mortlock ${ }^{45}$, D. Munshi ${ }^{73,51}$, A. Murphy ${ }^{67}$, P. Naselsky ${ }^{68,32}$, P. Natoli ${ }^{30,2,41}$, C. B. Netterfield ${ }^{15}$, H. U. Nørgaard-Nielsen ${ }^{13}$, F. Noviello ${ }^{48}$, D. Novikov ${ }^{45}$, I. Novikov $^{68}$, S. Osborne ${ }^{76}$, F. Pajot ${ }^{48}$, F. Pasian ${ }^{40}$, G. Patanchon ${ }^{3}$, O. Perdereau ${ }^{62}$, L. Perotto ${ }^{61}$, F. Perrotta ${ }^{71}$, F. Piacentini ${ }^{27}$, M. Piat ${ }^{3}$, E. Pierpaoli ${ }^{17}$, R. Piffaretti ${ }^{59,12}$, S. Plaszczynski ${ }^{62}$, E. Pointecouteau ${ }^{78,7}$, G. Polenta ${ }^{2,39}$, N. Ponthieu ${ }^{48}$, T. Poutanen ${ }^{37,19,1}$, G. W. Pratt ${ }^{59}$, G. Prézeau ${ }^{8,55}$, S. Prunet ${ }^{49}$, J.-L. Puget ${ }^{48}$, J. P. Rachen ${ }^{64}$, R. Rebolo ${ }^{53,33}$, M. Reinecke ${ }^{64}$, C. Renault ${ }^{61}$, S. Ricciardi ${ }^{41}$, T. Riller ${ }^{64}$, I. Ristorcelli ${ }^{78,7}$, G. Rocha $^{55,8}$, C. Rosset $^{3}$, J. A. Rubiño-Martín ${ }^{53,33}$, B. Rusholme ${ }^{46}$, M. Sandri ${ }^{41}$, D. Santos ${ }^{61}$, G. Savini ${ }^{70}$, B. M. Schaefer ${ }^{77}$, D. Scott ${ }^{16}$, M. D. Seiffert ${ }^{55,8}$, P. Shellard ${ }^{11}$, G. F. Smoot $22,63,3$, J.-L. Starck ${ }^{59,12}$, F. Stivoli ${ }^{43}$, V. Stolyarov ${ }^{4}$, R. Sudiwala ${ }^{73}$, R. Sunyaev ${ }^{64,74}$, J.-F. Sygnet ${ }^{49}$, J. A. Tauber ${ }^{36}$, L. Terenzi $^{41}$, L. Toffolatti ${ }^{14}$, M. Tomasi ${ }^{28,42}$, J.-P. Torre ${ }^{48}$, M. Tristram ${ }^{62}$, J. Tuovinen ${ }^{66}$, L. Valenziano $^{41}$, L. Vibert ${ }^{48}$, P. Vielva $^{54}$, F. Villa $^{41}$, N. Vittorio $^{31}$, L. A. Wade ${ }^{55}$, B. D. Wandelt ${ }^{49,25}$, S. D. M. White ${ }^{64}$, M. White ${ }^{22}$, D. Yvon ${ }^{12}$, A. Zacchei ${ }^{40}$, and A. Zonca ${ }^{24}$

\section{(Affiliations can be found after the references)}

Received 7 January 2011 / Accepted 5 May 2011

\section{ABSTRACT}

We present precise Sunyaev-Zeldovich (SZ) effect measurements in the direction of 62 nearby galaxy clusters $(z<0.5)$ detected at high signal-tonoise in the first Planck all-sky data set. The sample spans approximately a decade in total mass, $2 \times 10^{14} M_{\odot}<M_{500}<2 \times 10^{15} M_{\odot}$, where $M_{500}$ is the mass corresponding to a total density contrast of 500. Combining these high quality Planck measurements with deep XMM-Newton X-ray data, we investigate the relations between $D_{\mathrm{A}}^{2} Y_{500}$, the integrated Compton parameter due to the $\mathrm{SZ}$ effect, and the X-ray-derived gas mass $M_{\mathrm{g}, 500}$, temperature $T_{\mathrm{X}}$, luminosity $L_{\mathrm{X}, 500}$, SZ signal analogue $Y_{\mathrm{X}, 500}=M_{\mathrm{g}, 500} \times T_{\mathrm{X}}$, and total mass $M_{500}$. After correction for the effect of selection bias on the scaling relations, we find results that are in excellent agreement with both X-ray predictions and recently-published ground-based data derived from smaller samples. The present data yield an exceptionally robust, high-quality local reference, and illustrate Planck's unique capabilities for all-sky statistical studies of galaxy clusters.

Key words. galaxies: clusters: interacluster medium - X-rays: galaxies: clusters - cosmology: observations

\section{Introduction}

The X-ray emitting gas in galaxy clusters induces inverse Compton scattering of cosmic microwave background (CMB) photons, shifting their frequency distribution towards higher energies. First discussed in 1972 by Sunyaev \& Zeldovich, the scattering produces a characteristic distortion of the CMB spectrum in the direction of a cluster known as the thermal Sunyaev-

\footnotetext{
^ Corresponding author: G. W. Pratt, e-mail: gabriel.pratt@cea.fr
}

Zeldovich (SZ) effect. It is directly proportional to the Compton parameter $y$, a measure of the thermal electron pressure of the intracluster medium (ICM) gas along the line of sight: $y=$ $\left(\sigma_{\mathrm{T}} / m_{\mathrm{e}} c^{2}\right) \int P \mathrm{~d} l$. Here $P \propto n_{\mathrm{e}} T$ is the ICM thermal electron pressure, where $n_{\mathrm{e}}$ is the density and $T$ is the temperature, $\sigma_{\mathrm{T}}$ is the Thomson cross section, $m_{\mathrm{e}}$ is the electron rest mass, and $c$ is the speed of light. The SZ signal integrated over the cluster extent is proportional to the integrated Compton parameter, such 
that $D_{\mathrm{A}}^{2} Y_{\mathrm{SZ}}=\left(\sigma_{\mathrm{T}} / m_{\mathrm{e}} c^{2}\right) \int P \mathrm{~d} V$, where $D_{\mathrm{A}}$ is the angular distance to the source.

Clusters are currently thought to form via the hierarchical gravitational collapse of dark matter haloes, so that their number as a function of mass and redshift is a sensitive indicator of the underlying cosmology. The ICM is formed when gas falls into the dark matter gravitational potential and is heated to X-ray emitting temperatures by shocks and compression. The scalefree nature of this process implies that simple power law relationships exist between the total halo mass and various other physical properties (e.g., Bertschinger 1985; Kaiser 1986) such as X-ray temperature $T$ or luminosity $L$ (e.g., Voit 2005; Arnaud et al. 2005, 2007; Pratt et al. 2009; Vikhlinin et al. 2009). As the total mass is not directly observable, such mass proxies are needed to leverage the statistical power of various large-scale surveys for cosmological applications. Since the gas pressure is directly related to the depth of the gravitational potential, the quantity $D_{\mathrm{A}}^{2} Y_{\mathrm{SZ}}$ is expected to scale particularly closely with the total mass, a claim supported by recent numerical simulations (e.g., White et al. 2002; da Silva et al. 2004; Motl et al. 2005; Nagai 2006; Wik et al. 2008; Aghanim et al. 2009). SZ surveys for galaxy clusters thus have great potential to produce competitive cosmological constraints.

In a few short years, SZ observations have progressed from the first spatially resolved observations of individual objects (Pointecouteau et al. 1999, 2001; Komatsu et al. 1999, 2001), to the first discoveries of new objects (Staniszewski et al. 2009), to large-scale survey projects for cosmology such as the Atacama Cosmology Telescope (ACT, Kosowsky 2003) and the South Pole Telescope (SPT, Carlstrom et al. 2011). Indeed, first cosmological results from these surveys have started appearing (Vanderlinde et al. 2010; Sehgal et al. 2011). Attention is now focussing on the shape and normalisation of the pressure profile (e.g., Nagai et al. 2007; Arnaud et al. 2010; Komatsu et al. 2011), calibration of the relationship between $D_{\mathrm{A}}^{2} Y_{\mathrm{SZ}}$ and the total mass for cosmological applications (e.g., Bonamente et al. 2008; Marrone et al. 2009; Arnaud et al. 2010; Melin et al. 2011), comparison of the measured SZ signal to X-ray predictions (Lieu et al. 2006; Bielby \& Shanks 2007; Afshordi et al. 2007; Komatsu et al. 2011; Melin et al. 2011), and the relationship between $D_{\mathrm{A}}^{2} Y_{\mathrm{SZ}}$ and its X-ray analogue $Y_{\mathrm{X}, 500}$ (e.g., Andersson et al. 2011). First introduced by Kravtsov et al. (2006), the latter quantity is defined as the product of $M_{\mathrm{g}}$, the gas mass, and $T_{\mathrm{X}}$, the spectroscopic temperature excluding the core regions. As the link between $Y_{\mathrm{X}, 500}$ and $D_{\mathrm{A}}^{2} Y_{\mathrm{SZ}}$ depends on the relationship between the gas mass weighted and $\mathrm{X}$-ray spectroscopic temperatures, it is a sensitive probe of cluster astrophysics.

In the following, we use a subsample of Planck ${ }^{1}$ Early Release Compact Source Catalogue SZ (ESZ) clusters, consisting of high signal-to-noise ratio $(\mathrm{S} / \mathrm{N})$ Planck detections with deep XMM-Newton archive observations, to investigate the local $(z \lesssim 0.5)$ SZ scaling relations. Given its all-sky coverage and high sensitivity, Planck is uniquely suited to this task, allowing high $\mathrm{S} / \mathrm{N}$ detection of many hot, massive systems that do not appear in other SZ surveys due simply to their limited area; correspondingly, the large field of view and collecting power of XMM-Newton make it the ideal instrument to

\footnotetext{
1 Planck (http://www.esa.int/Planck) is a project of the European Space Agency (ESA) with instruments provided by two scientific consortia funded by ESA member states (in particular the lead countries France and Italy), with contributions from NASA (USA) and telescope reflectors provided by a collaboration between ESA and a scientific consortium led and funded by Denmark.
}

observe these objects in X-rays out to a significant fraction of the virial radius. Here we investigate the relationship between SZ quantities and X-ray quantities, making full use of the exceptional quality of both data sets. Two complementary companion papers (Planck Collaboration 2011f,h) harness the statistical power of the Planck survey by analysing the SZ flux-X-ray luminosity and SZ flux-optical richness relations, respectively, using a bin-averaging approach. Two further companion papers present the parent catalogue (Planck Collaboration 2011d) and XMM-Newton validation observations of newly-discovered clusters (Planck Collaboration 2011e).

In this paper we adopt a $\Lambda$ CDM cosmology with $H_{0}=$ $70 \mathrm{~km} \mathrm{~s}^{-1} \mathrm{Mpc}^{-1}, \Omega_{\mathrm{M}}=0.3$ and $\Omega_{\Lambda}=0.7$. The factor $E(z)=$ $\sqrt{\Omega_{\mathrm{M}}(1+z)^{3}+\Omega_{\Lambda}}$ is the ratio of the Hubble constant at redshift $z$ to its present day value. The variables $M_{500}$ and $R_{500}$ are the total mass and radius corresponding to a total density contrast $500 \rho_{\mathrm{c}}(z)$, where $\rho_{\mathrm{c}}(z)$ is the critical density of the Universe at the cluster redshift; thus $M_{500}=(4 \pi / 3) 500 \rho_{\mathrm{c}}(z) R_{500}^{3}$. The quantity $Y_{\mathrm{X}, 500}$ is defined as the product of $M_{\mathrm{g}, 500}$, the gas mass within $R_{500}$, and $T_{\mathrm{X}}$, the spectroscopic temperature measured in the [0.15-0.75] $R_{500}$ aperture. The SZ signal is denoted $Y_{500}$ throughout. This quantity is defined by $D_{\mathrm{A}}^{2} Y_{500} \equiv$ $\left(\sigma_{\mathrm{T}} / m_{\mathrm{e}} c^{2}\right) \int P \mathrm{~d} V$. Here $D_{\mathrm{A}}$ is the angular distance to the system, $\sigma_{\mathrm{T}}$ is the Thomson cross-section, $c$ the speed of light, $m_{\mathrm{e}}$ the electron rest mass, $P \propto n_{\mathrm{e}} T$ is the pressure (the product of the electron number density and temperature), and the integration is performed over a sphere of radius $R_{500}$. The quantity $D_{\mathrm{A}}^{2} Y_{500}$ is the spherically integrated Compton parameter, and $Y_{500}$ is proportional to the flux of the SZ signal within $R_{500}$.

\section{The ESZ catalogue and the Planck XMM-Newton archive subsample}

\subsection{Planck and the ESZ catalogue}

Planck (Tauber et al. 2010; Planck Collaboration 2011a) is the third generation space mission to measure the anisotropy of the CMB. It observes the sky in nine frequency bands covering 30$857 \mathrm{GHz}$ with high sensitivity and angular resolution from 31' to $5^{\prime}$. The Low Frequency Instrument (LFI; Mandolesi et al. 2010; Bersanelli et al. 2010; Mennella et al. 2011) covers the 30,44 , and $70 \mathrm{GHz}$ bands with amplifiers cooled to $20 \mathrm{~K}$. The High Frequency Instrument (HFI; Lamarre et al. 2010; Planck HFI Core Team 2011a) covers the 100, 143, 217, 353, 545, and $857 \mathrm{GHz}$ bands with bolometers cooled to $0.1 \mathrm{~K}$. Polarization is measured in all but the highest two bands (Leahy et al. 2010; Rosset et al. 2010). A combination of radiative cooling and three mechanical coolers produces the temperatures needed for the detectors and optics (Planck Collaboration 2011b). Two Data Processing Centres (DPCs) check and calibrate the data and make maps of the sky (Planck HFI Core Team 2011b; Zacchei et al. 2011). Planck's sensitivity, angular resolution, and frequency coverage make it a powerful instrument for galactic and extragalactic astrophysics as well as cosmology. Early astrophysics results are given in Planck Collaboration (2011e-u) .

The basic data set used in the present paper is the ESZ sample, described in detail in Planck Collaboration (2011d). The sample is derived from the highest $\mathrm{S} / \mathrm{N}$ detections $(\mathrm{S} / \mathrm{N}>6)$ in a blind multi-frequency search in the all-sky maps from observations obtained in the first ten months of the Planck mission. 


\subsection{The Planck-XMM-Newton archive subsample}

Cross-correlation of the ESZ subsample with the Meta Catalogue of X-ray Clusters (MCXC; Piffaretti et al. 2011) produced 158 matches with known X-ray clusters. As shown in Fig. 1, these objects lie at a redshift $z \lesssim 0.5$ and cover approximately a decade in mass ${ }^{2}$. A search for these clusters in the XMM-Newton Science Archive ${ }^{3}$ produced a combined Planck$X M M$-Newton archive sample of 88 objects as of July 2010, indicated by blue crosses in Fig. 1.

As detailed below in Sect. 3, we used different X-ray data processing techniques depending on cluster angular extent. More specifically, if the source extent lies well within the XMM-Newton field of view then the X-ray background can be characterised using a source-free region of the observation, while clusters with a larger angular extent require simultaneous source and background modelling. We label these classes of clusters as A and B, respectively. The dashed line in Fig. 1 illustrates the radius at which $R_{500} \lesssim 12^{\prime}$, corresponding to the maximum angular extent within which the X-ray background can be characterised in a single XMM-Newton field of view. Using this criterion, we divide the Planck-XMM-Newton archive sample into 58 A clusters and 30 B clusters.

Not all of the clusters in the full Planck-XMM-Newton archive sample are used in the present paper. Some observations in the A cluster list were excluded because soft proton solar flare contamination had rendered the observations unusable, or because the object had not yet been observed at the time of the archive search, or because the target was a clear multiple system unsuited to a spherically-symmetric analysis. For the B clusters, in addition to the high-luminosity systems already published in Bourdin \& Mazzotta (2008), we prioritised those where the XMM-Newton field of view was expected to cover the largest possible fraction of $R_{500}$, corresponding to objects with the lowest estimated mass in Fig. 1. The final sample of 62 systems consists of $44 \mathrm{~A}$ objects and $18 \mathrm{~B}$ objects. While the sample is neither representative nor complete, it represents the largest, highest-quality SZ-X-ray data set currently-available.

\section{X-ray cluster properties}

\subsection{X-ray data processing: A clusters}

For the A clusters, we use the latest version (v10.0) of the $X M M-N e w t o n-S A S$, ensuring that the most recent calibration corrections are applied to the X-ray photons. Event lists are processed, cleaned for periods of high background, PATTERN-selected and corrected for vignetting as detailed in Pratt et al. (2007).

The quiescent XMM-Newton background is dominated by events due to charged particles. We subtract this component by using a background file built from stacked observations obtained with the filter wheel in the CLOSED position, recast to the source position and renormalised using the count rate in the high energy band free of cluster emission ${ }^{4}$. The remaining components are due to the cosmic X-ray background produced by unresolved sources and a diffuse soft X-ray contribution attributable to the Galaxy. The A sample is selected so that $R_{500} \lesssim 12^{\prime}$, allowing us to model these remaining components using emission

\footnotetext{
2 Estimated from the X-ray luminosity-mass relation of Pratt et al. (2009), as detailed in Piffaretti et al. (2011).

3 http://xmm. esac.esa.int/xsa/

4 We excluded a $5^{\prime}$ region around the cluster centre to avoid contamination from residual cluster emission.
}

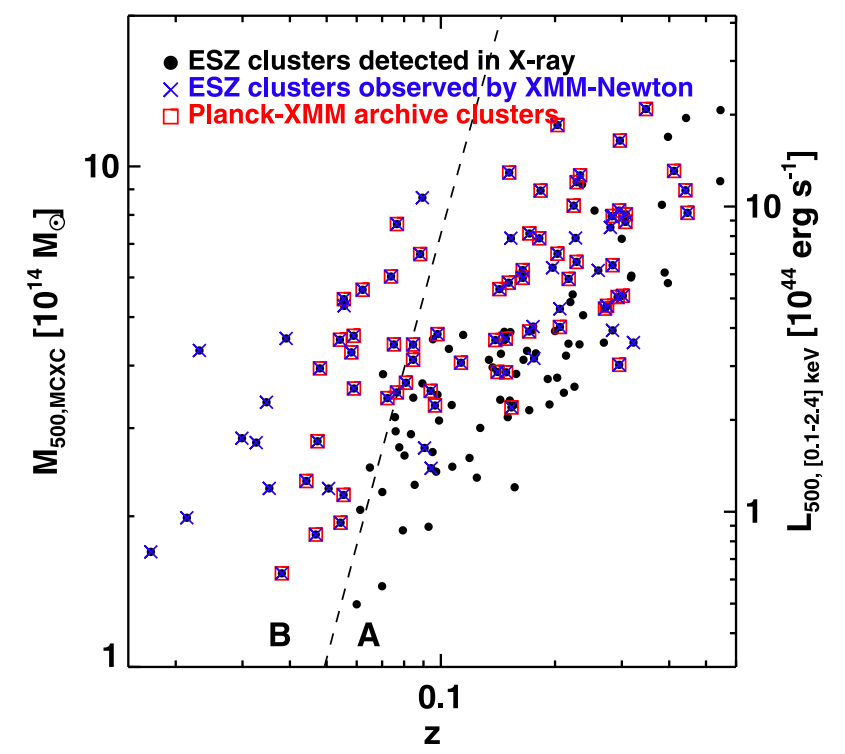

Fig. 1. The 158 Planck ESZ clusters already observed in X-rays. Masses are estimated from their X-ray luminosity as described in Piffaretti et al. (2011). The 62 clusters analysed and discussed in this paper are indicated by the red squares. The dashed line represents the locus at which $R_{500} \sim 12^{\prime}$.

from an annular region external to the cluster emission as detailed in Croston et al. (2008) and Pratt et al. (2010).

Point sources were identified from the small scales of wavelet-decomposed images in the [0.3-2] and [2-5] keV bands. These sources were excluded, with the exclusion radius matched to the variation of the PSF size across the detector. We also masked any well-defined substructures that produced prominent secondary maxima and were visible in the larger scales of the wavelet decomposition process.

Surface brightness profiles were extracted from the data in 3". 3 bins centred on the X-ray peak. Finally, a non-parametric regularisation method was used to derive the deprojected, PSFcorrected density profiles, $n_{\mathrm{e}}(r)$, as described in Croston et al. (2008).

\subsection{X-ray data processing: B clusters}

For each object in the B cluster sample, a merged energyposition photon cube was built from the various observations of a given target. The cube was built from soft proton-cleaned events from each camera, generated with v10.0 of the XMM-NewtonSAS, to which an effective exposure and a background noise array were added. The exposure array was computed from the effective exposure time, with corrections for spatially variable mirror effective areas, filter transmissions, CCD pixel area, chip gaps and bad pixels, as appropriate. The background noise array was modelled as the sum of components accounting for the Galactic foreground and cosmic X-ray background, plus charged particle-induced and out-of-time events. Full details of the method are given in Bourdin \& Mazzotta (2008).

The Galactic foreground is a critical model component in the case of the B clusters. These objects often extend over the full XMM-Newton field of view, so that the cluster emission cannot be spatially separated from the foreground components. We thus constrained the foreground components using a joint fit of cluster emissivity and temperature in an external annulus corresponding to $\sim R_{500}$. Despite the degeneracy of this estimate with the cluster emissivity itself, in all cases the temperature 

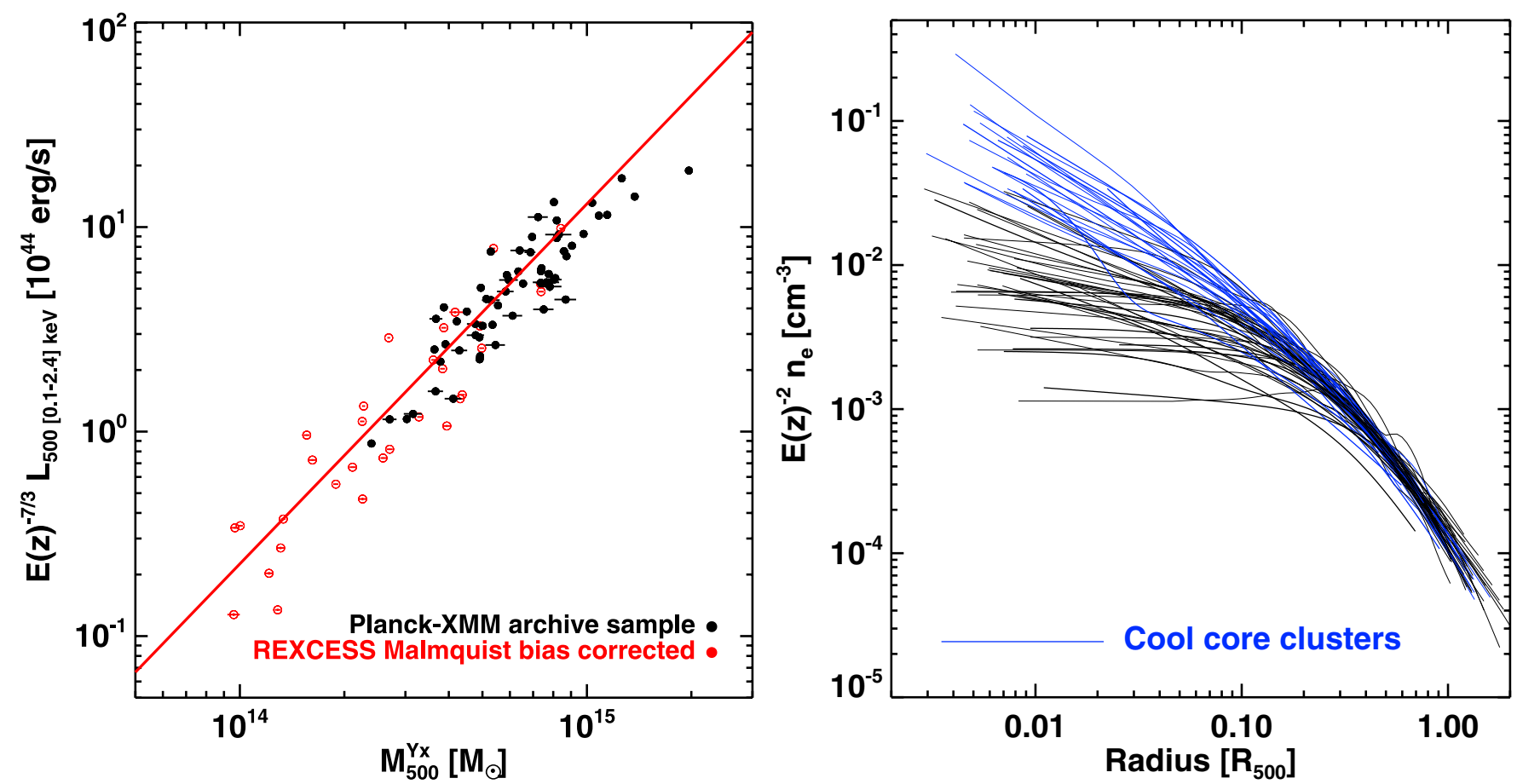

Fig. 2. Left: $L_{\mathrm{X}, 500}-M_{500}$ relation of the Planck-XMM-Newton archive sample compared to REXCESS, a representative X-ray cluster sample. Luminosity is estimated interior to $R_{500}$ in the [0.1-2.4] keV band, and mass from the $M_{500}-Y_{\mathrm{X}, 500}$ relation of Arnaud et al. (2010). The solid red line is the fit to the REXCESS sample only (Pratt et al. 2009). Right: scaled density profiles of the 62 systems in the Planck-XMM-Newton archive sample. Profiles have been corrected for projection and PSF effects as described in the text. Systems classified as cool cores are indicated by blue lines.

obtained in this annulus was found to be lower than the average cluster temperature, as is commonly observed in clusters allowing full cluster-foreground spatial separation (e.g., Pratt et al. 2007; Leccardi \& Molendi 2008).

The ICM density profiles in the B cluster sample were then derived using the analytic distributions of ICM density and temperature introduced by Vikhlinin et al. (2006). These parametric distributions were projected along the line of sight, convolved with the XMM-Newton PSF, and fitted to the observed projected cluster brightness and temperature profiles. The resulting density profiles, $n_{\mathrm{e}}(r)$, were used to derive X-ray quantities for each cluster as described below.

\subsection{X-ray quantities}

For the current generation of high-resolution X-ray telescopes, the effective limiting radius for high-quality nearby observations of the type discussed here is $R_{500}$. Beyond this radius, the effect of the variable background becomes dominant and the uncertainties begin to become difficult to quantify. In addition, as shown by Evrard et al. (1996), $R_{500}$ is also the radius within which clusters are relatively relaxed. We estimate the X-ray quantities for each cluster self-consistently within $R_{500}$ using the $M_{500}-Y_{\mathrm{X}, 500}$ relation given in Arnaud et al. (2010, see also Pratt et al. 2010), assuming standard evolution, viz.,

$E(z)^{2 / 5} M_{500}=10^{14.567 \pm 0.010}\left[\frac{Y_{\mathrm{X}, 500}}{2 \times 10^{14} M_{\odot} \mathrm{keV}}\right]^{0.561 \pm 0.018} M_{\odot}$

The radius $R_{500}$ was calculated iteratively as described in Kravtsov et al. (2006). Using Eq. (1) and the definition of $M_{500}$ and $Y_{\mathrm{X}, 500}$, an equation of the form $R_{500}^{3}=C\left[M_{\mathrm{g}, 500} T_{\mathrm{X}}\right]^{\alpha}$ must be solved. Starting from an initial temperature measurement, the equation is solved for $R_{500}$, with the gas mass computed from the density profiles discussed above in Sects. 3.1 and 3.2. A new temperature is then estimated within $[0.15-0.75] R_{500}$ and the procedure is repeated until convergence. Once converged, we measure the temperature within an aperture corresponding to $R_{500}$ and calculate the [0.1-2.4] keV and [0.5-2] keV band luminosities as described in Pratt et al. (2009). The resulting X-ray quantities are listed in Table 1.

The left-hand panel of Fig. 2 shows the $L_{X, 500}-M_{500}$ relation of the Planck-XMM-Newton archive sample, where $L_{\mathrm{X}, 500}$ is the $\mathrm{X}$-ray luminosity estimated interior to $R_{500}$ in the $[0.1-2.4] \mathrm{keV}$ band ${ }^{5}$, and the mass is estimated from the $M_{500}-Y_{\mathrm{X}, 500}$ relation given in Eq. (1). The data are compared to the equivalent relation from REXCESS, a sample designed to be representative of the X-ray cluster population (Böhringer et al. 2007). One can see that the Planck-XMM-Newton archive clusters are all massive, luminous systems, as expected for objects detected in SZ at high $\mathrm{S} / \mathrm{N}$ by Planck. They follow the general trend exhibited by REXCESS (Pratt et al. 2009), but extend to higher mass and luminosity.

\subsection{Scaled gas density profiles and cool core subsample}

The scaled gas density profiles of the full sample of 62 clusters are shown in the right-hand panel of Fig. 2, where each profile

\footnotetext{
5 The standard [0.1-2.4] $\mathrm{keV}$ band self-similar evolution factor is $E(z)^{-2}$. However, the observed scaling relations are steeper than selfsimilar (see e.g., Pratt et al. 2009), leading to a dependence of the evolution factor on the quantity to which it is applied. In the present work we use an evolution factor of $E(z)^{-7 / 3}$, appropriate for bolometric luminosities, for comparison to REXCESS (see also Maughan 2007).
} 
has been corrected for evolution and scaled to $R_{500}$. As has been seen in other cluster samples (e.g., Croston et al. 2008), there is a large amount of scatter in the central regions, extending out to $\sim 0.15 R_{500}$, beyond which the profiles rapidly converge.

It is well-known that some clusters exhibit so-called cool cores, central regions of very dense gas where the cooling time is less than the Hubble time (e.g., Jones \& Forman 1984). Such objects have very high X-ray luminosities and extremely low central entropies that tend to set them apart from the rest of the X-ray cluster population (e.g., Fabian et al. 1994; Pratt et al. 2010). In addition, the current consensus is that these systems represent a generally more relaxed subset of the cluster population (although see Burns et al. 2008 for a dissenting view). Following Pratt et al. (2009), we estimated the central gas density $n_{\mathrm{e}, 0}$ using a $\beta$ model fit to the gas density profile interior to $0.05 R_{500}$, and classified objects with $E(z)^{-2} n_{\mathrm{e}, 0}>4 \times 10^{-2} \mathrm{~cm}^{-3}$ as cool core systems. In total, 22/62 clusters in the present sample are classified as such. These are plotted in blue in Fig. 2 and in all following plots.

\section{SZ cluster properties}

\subsection{Optimisation of the $S Z$ flux extraction}

The basic SZ signal extraction procedure is described in full in Planck Collaboration (2011d). In brief, this procedure consists of applying multi-frequency matched filters (MMF, Melin et al. 2006), that incorporate prior knowledge of the signal, to the Planck maps. Specifically, the ICM pressure is assumed to follow the universal profile shape derived from the REXCESS sample by Arnaud et al. (2010). The SZ flux is computed by integrating along the line-of-sight and normalising the universal pressure profile. Each profile is truncated at $5 \times R_{500}$, effectively giving a measure of the flux within a cylinder of aperture radius $5 \times R_{500}$, and then converted to the value in a sphere of radius $R_{500}$ for direct comparison with the X-ray prediction. This is the fundamental SZ quantity used in the present paper $^{6}$, and we refer to it throughout as $Y_{500}$.

Section 6 of Planck Collaboration (2011d) shows that the cluster flux derived from blind application of the MMF algorithm is systematically larger than X-ray expectations. This discrepancy is a result of overestimation of the cluster size $\theta_{500}$ due to the freedom to optimise significance with position and size. As shown in Planck Collaboration (2011d), if the SZ signal is instead extracted from a region centred on the X-ray position with size $\theta_{500}$ estimated from the X-ray luminosity-mass relation, the SZ flux is in better agreement with X-ray expectations. When additional constraints on the cluster size are available, the SZ flux extraction can be further optimised.

With the present cluster sample we can make use of the higher-quality estimate of the X-ray size $\theta_{500}$, derived from $R_{500}$, measured using the $M_{500}-Y_{\mathrm{X}, 500}$ relation as detailed in Sect. 3.3. Appendix A details the improvement in SZ flux extraction when these higher-quality size estimates are used. For each cluster in the sample, we thus re-ran the SZ flux extraction, calculating $Y_{500}$ with the X-ray position and size fixed to the refined values derived from the high-quality XMM-Newton observation.

\subsection{Robustness tests specific to local sample}

Section 6 of Planck Collaboration (2011d) details various robustness tests relevant to all Planck SZ papers, including

\footnotetext{
${ }^{6}$ Note that $Y_{500}$ is the directly observed "apparent" quantity, while $D_{\AA}^{2} Y_{500}$ is the corresponding "absolute" quantity, intrinsic to the cluster.
}

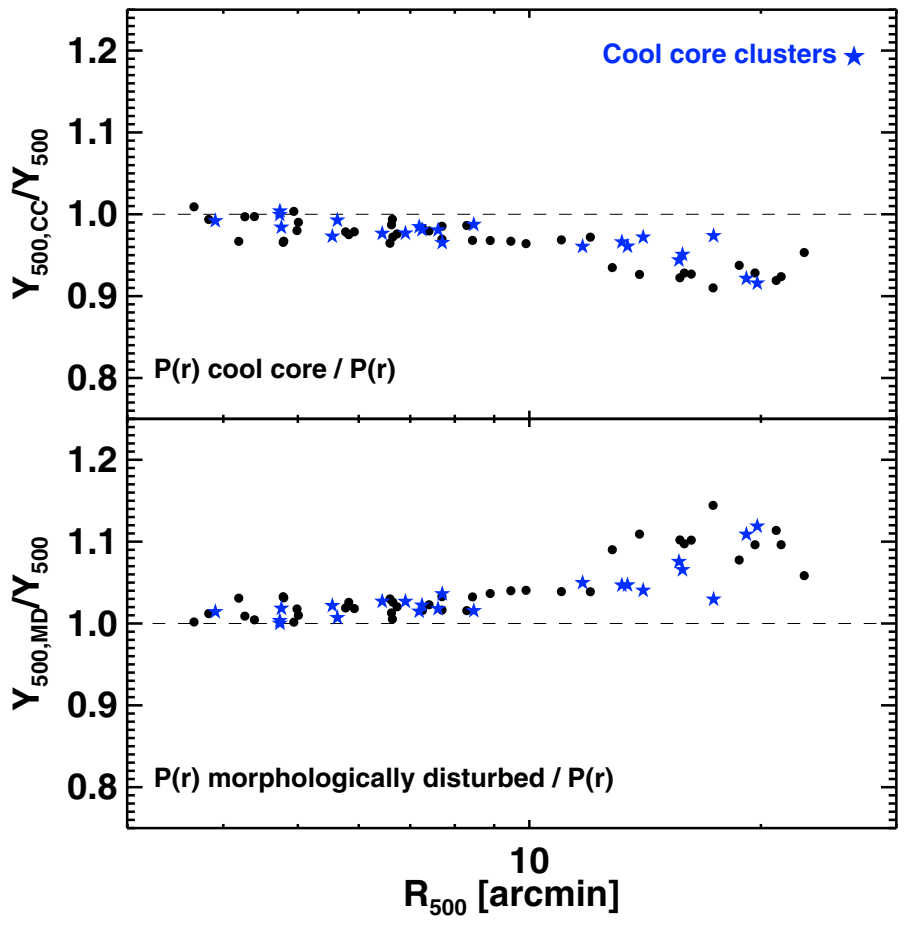

Fig. 3. Comparison of $Y_{500}$ from extraction using the baseline pressure profile with that from the best fitting REXCESS cool core and morphologically disturbed pressure profiles given in Appendix C of Arnaud et al. (2010). Cool core systems are marked as blue stars, other systems as black dots. Top: universal pressure profile vs. cool-core pressure profile; Bottom: universal pressure profile vs. morphologically-disturbed pressure profile. The trend with $R_{500}$ is due to the inability of the Planck beam to resolve different profiles at small angular size. The effect is small (maximum $\sim 10$ per cent) and quasi-symmetric (as expected), so no bias is introduced.

investigation of the cluster size-flux degeneracy discussed above, the impact of the assumed pressure profile used for cluster detection, beam-shape effects, colour corrections, contamination by point sources, and discussion of the overall error budget. For the present sample we undertake two further robustness tests: the first is related to the impact of radio source contamination; the second examines the impact of the assumed pressure profile shape on the derived $Y_{500}$.

\subsubsection{Contamination by point sources}

Contamination by point sources can affect extraction of the SZ parameters, and have implications for astrophysical studies of clusters or further cosmological applications (Aghanim et al. 2005; Douspis et al. 2006). We have thus checked the possible effect of radio galaxies on the derived $Y_{500}$ by combining data from SUMSS (Bock et al. 1999, a catalogue of radio sources at $0.85 \mathrm{GHz}$ ), NVSS (Condon et al. 1998, a catalogue of radio sources at $1.4 \mathrm{GHz}$ ), and data from the Planck LFI and HFI. Two clusters in our sample exhibit relatively bright $(S(1.4 \mathrm{GHz}) \gtrsim 1 \mathrm{Jy})$, flat spectrum radio sources within a radius of $15^{\prime}$ from the X-ray peak. These sources are clearly seen in LFI data and could potentially affect the SZ measurement. However, as we discuss below in Sect. 5, inclusion or exclusion of these objects has a negligible effect on the derived scaling relations. 
Table 1. X-ray and SZ properties.

\begin{tabular}{|c|c|c|c|c|c|c|c|c|c|c|c|}
\hline Name & $\begin{array}{c}\text { RA } \\
{[\operatorname{deg}]}\end{array}$ & $\begin{array}{c}\text { Dec } \\
{[\mathrm{deg}]}\end{array}$ & $z$ & $\begin{array}{l}R_{500} \\
{[\mathrm{kpc}]}\end{array}$ & $\begin{array}{c}T_{\mathrm{X}} \\
{[\mathrm{keV}]}\end{array}$ & $\begin{array}{c}M_{\mathrm{g}, 500} \\
{\left[10^{14} M_{\odot}\right]}\end{array}$ & $\begin{array}{c}Y_{X, 500} \\
{\left[10^{14} M_{\odot} \mathrm{keV}\right]}\end{array}$ & $\begin{array}{c}D_{\mathrm{A}}^{2} Y_{500} \\
{\left[10^{-4} \mathrm{Mpc}^{2}\right]}\end{array}$ & $\begin{array}{c}M_{500} \\
{\left[10^{14} M_{\odot}\right]}\end{array}$ & $\begin{array}{c}L_{\mathrm{X}, 500} \\
{\left[10^{44} \mathrm{erg} \mathrm{s}^{-1}\right]}\end{array}$ & $\mathrm{CC}$ \\
\hline RXC J0014.3-3022 & 3.58 & -30.38 & 0.307 & 1358 & $7.72 \pm 0.25$ & $1.65 \pm 0.01$ & $12.73 \pm 0.51$ & $1.74 \pm 0.21$ & $9.78 \pm 0.21$ & $13.35 \pm 0.09$ & \\
\hline A85 & 10.44 & -9.37 & 0.052 & 1206 & $5.78 \pm 0.22$ & $0.66 \pm 0.01$ & $3.84 \pm 0.19$ & $0.47 \pm 0.05$ & $5.30 \pm 0.31$ & $4.65 \pm 0.02$ & $\checkmark$ \\
\hline RXC J0043.4-2037 & 10.84 & -20.61 & 0.292 & 1152 & $5.82 \pm 0.20$ & $0.88 \pm 0.01$ & $5.10 \pm 0.20$ & $1.40 \pm 0.17$ & $5.88 \pm 0.14$ & $8.26 \pm 0.08$ & \\
\hline A119 & 14.02 & -1.30 & 0.044 & 1114 & $5.40 \pm 0.23$ & $0.45 \pm 0.01$ & $2.45 \pm 0.14$ & $0.27 \pm 0.03$ & $4.12 \pm 0.23$ & $1.52 \pm 0.01$ & \\
\hline RXC J0232.2-4420 & 38.06 & -44.37 & 0.284 & 1223 & $6.41 \pm 0.20$ & $1.07 \pm 0.01$ & $6.86 \pm 0.26$ & $0.86 \pm 0.13$ & $6.95 \pm 0.15$ & $12.53 \pm 0.09$ & $\checkmark$ \\
\hline A 401 & 44.73 & 13.56 & 0.075 & 1355 & $7.26 \pm 0.44$ & $1.02 \pm 0.04$ & $7.43 \pm 0.58$ & $0.83 \pm 0.08$ & $7.65 \pm 0.67$ & $5.82 \pm 0.04$ & \\
\hline RXC J0303.8-7752 & 46.00 & -77.88 & 0.274 & 1251 & $7.88 \pm 0.36$ & $0.96 \pm 0.02$ & $7.58 \pm 0.45$ & $1.09 \pm 0.13$ & $7.37 \pm 0.25$ & $7.39 \pm 0.07$ & \\
\hline A 3112 & 49.51 & -44.26 & 0.070 & 1062 & $5.02 \pm 0.15$ & $0.40 \pm 0.01$ & $2.03 \pm 0.07$ & $0.18 \pm 0.03$ & $3.67 \pm 0.16$ & $3.84 \pm 0.02$ & $\checkmark$ \\
\hline A3158 & 55.72 & -53.60 & 0.060 & 1124 & $5.00 \pm 0.18$ & $0.53 \pm 0.01$ & $2.66 \pm 0.12$ & $0.35 \pm 0.03$ & $4.29 \pm 0.23$ & $2.66 \pm 0.01$ & \\
\hline A478 & 63.35 & 10.45 & 0.088 & 1326 & $6.43 \pm 0.19$ & $1.06 \pm 0.03$ & $6.81 \pm 0.26$ & $0.92 \pm 0.08$ & $7.23 \pm 0.48$ & $12.33 \pm 0.05$ & $\checkmark$ \\
\hline A3266 & 67.83 & -61.42 & 0.059 & 1354 & $7.46 \pm 0.22$ & $0.96 \pm 0.02$ & $7.17 \pm 0.30$ & $0.90 \pm 0.07$ & $7.51 \pm 0.51$ & $4.22 \pm 0.01$ & \\
\hline A520 & 73.55 & 2.96 & 0.203 & 1325 & $7.74 \pm 0.22$ & $1.13 \pm 0.01$ & $8.75=$ & $0.99 \pm 0.14$ & $8.11 \pm 0.16$ & 0.04 & \\
\hline $\mathrm{XCJ}$ & 79.17 & -54.52 & 0.295 & 1266 & $.11 \pm 0.67$ & $1.20 \pm 0.06$ & 06 & $1.29 \pm$ & $7.82 \pm 0.60$ & 7.27 & \\
\hline C J0528.9-3927 & 82.22 & -39.44 & 0.284 & 1218 & $6.04 \pm 0.32$ & $1.11 \pm 0.02$ & 46 & $1.18 \pm$ & .25 & 10.55 & $\checkmark$ \\
\hline RXC J0532.9-3701 & 83.23 & -37.02 & 0.275 & 1190 & $6.84 \pm$ & $0.85 \pm 0$ & & $0.97 \pm$ & 17 & .07 & $\checkmark$ \\
\hline RXC J0547.6-3152 & 39 & -31.90 & 0.148 & 1150 & 6.10 & $0.60 \pm$ & & 0.4 & & 02 & \\
\hline A3376 & 90.47 & -39 & 0.045 & 930 & $3.39 \pm$ & $0.28 \pm 0.01$ & 33 & $0.10 \pm$ & 06 & 01 & \\
\hline RXC J0605.8-3518 & 91.48 & -35.29 & 0.139 & 1059 & $4.93 \pm$ & $0.46 \pm 0.01$ & 2.2 & $0.47 \pm$ & 06 & 4.74 & $\checkmark$ \\
\hline RXC J0 & 101.39 & -54.21 & 0.164 & 1303 & $7.26 \pm 0.18$ & $1.01 \pm 0.01$ & 7.33 & $1.09 \pm$ & 7.40 & 7.59 & $\checkmark$ \\
\hline RXC J06 & 4.63 & -55.96 & 0.296 & 1527 & $11.19 \pm 0.25$ & $2.08 \pm 0.02$ & $23.22=$ & $2.66 \pm$ & 13.73 & 20.05 & \\
\hline A665 & 127.75 & 65.88 & 0.182 & 1331 & $7.64 \pm 0.46$ & $1.12 \pm 0.03$ & 61 & $1.09 \pm($ & $8.04 \pm$ & 6.8 & \\
\hline A754 & 137.24 & -9.65 & 0.054 & 1423 & $8.93 \pm 0.24$ & $1.04 \pm 0.03$ & $9.28 \pm 0.39$ & $0.86 \pm 0.05$ & $8.69 \pm 0.63$ & 4.68 & \\
\hline A773 & 139.49 & 51.69 & 0.217 & 1228 & $6.78 \pm 0.16$ & $0.89 \pm 0.01$ & $6.01 \pm 0.18$ & $0.86 \pm 0.11$ & $6.55 \pm 0.11$ & $6.80=$ & \\
\hline A781 & 140.09 & 30.49 & 0.298 & 1114 & $5.72 \pm 0.10$ & $0.76 \pm 0.01$ & $4.32=$ & $0.72 \pm 0.14$ & $5.35 \pm 0.07$ & 4.75 & \\
\hline A868 & 6.36 & -8.64 & 0.153 & 1058 & $4.63 \pm 0.16$ & $0.51 \pm 0.01$ & 08 & $0.41 \pm$ & $3.91 \pm 0.10$ & 3.18 & \\
\hline A963 & 4.24 & 39.01 & 0.206 & 1123 & $9 \pm 0.11$ & $0.66 \pm 0.01$ & 09 & $0.41 \pm$ & $4.95=$ & 03 & $\checkmark$ \\
\hline RXC J1131 & 3.00 & -19.92 & 0.308 & 1300 & $7.75 \pm 0.31$ & $1.30 \pm 0.02$ & 53 & $1.30 \pm$ & 8.59 & 11.0 & \\
\hline 413 & 1 & 23.39 & 0.143 & 1242 & 7 & $0.82 \pm 0$ & & & & & $\checkmark$ \\
\hline J1206.2-0848 & 59 & -8.81 & 0.441 & 1334 & $10.15 \pm$ & $1.59 \pm c$ & 63 & $1.70 \pm$ & 10.8 & 19. & $\checkmark$ \\
\hline ZwCl1215 & & & 0.077 & 1211 & 7 & $0.63 \pm$ & & 77 & & & \\
\hline A1 & & 63 & 0.302 & 1145 & 7 & $0.80 \pm$ & & 1 & & 18 & \\
\hline $528 \mathrm{~S}$ & .65 & -29.21 & 0.053 & 966 & 1 & $0.28 \pm 0.01$ & & 3 & 2.7 & 1.2 & $\checkmark$ \\
\hline 544 & 4.30 & -17.40 & 0.047 & 1070 & 20 & $0.41 \pm 0$ & 1 & $0.25=$ & 3. & 1.6 & $\checkmark$ \\
\hline A3532 & 4.39 & -30.41 & 0.056 & 1015 & $4.44 \pm 0.30$ & $0.34 \pm 0.01$ & 53 & $0.21 \pm$ & 3.1 & 1.30 & \\
\hline A1650 & 4.67 & -1.76 & 0.084 & 1110 & $5.11 \pm 0.06$ & $0.51 \pm 0.01$ & $2.61=$ & $0.44 \pm 0.06$ & $4.22 \pm 0.03$ & 3.79 & $\checkmark$ \\
\hline A1651 & 194.88 & -4.20 & 0.084 & 1135 & $5.23 \pm 0.12$ & $0.56 \pm 0.01$ & $2.94 \pm 0.08$ & $0.36 \pm 0.06$ & $4.51 \pm 0.07$ & $4.23 \pm 0.02$ & \\
\hline A1689 & 7.88 & -1.35 & 0.183 & 1339 & $8.17 \pm 0.12$ & $1.08 \pm 0.01$ & $8.84 \pm 0.15$ & $1.37 \pm 0.15$ & $8.19 \pm 0.08$ & $13.29 \pm 0.03$ & $\checkmark$ \\
\hline A3558 & 2.00 & -31.51 & 0.047 & 1170 & $4.78 \pm 0.13$ & $0.67 \pm 0.02$ & $21=$ & $0.42 \pm 0.05$ & $4.77 \pm 0.26$ & 3.54 & \\
\hline A1763 & 3.80 & 41.00 & 0.223 & 1275 & $6.55 \pm 0.17$ & $1.14 \pm 0.01$ & $7.44 \pm 0.27$ & $1.28 \pm 0.12$ & $7.37 \pm 0.15$ & $8.00 \pm$ & \\
\hline A1795 & 7.24 & 26.58 & 0.062 & 1254 & $60 \pm 0.21$ & $0.73 \pm 0.02$ & $4.79=$ & $0.46 \pm$ & $5.96 \pm 0.37$ & 5.90 & 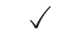 \\
\hline A1914 & 6.49 & 37.83 & 0.171 & 1345 & $.26 \pm 0.19$ & $1.07 \pm 0.01$ & $80 \pm 0.26$ & $1.00 \pm 0.09$ & $8.19 \pm 0.13$ & $10.73 \pm 0.05$ & \\
\hline A2034 & 7.53 & 33.49 & 0.151 & 1330 & $1 \pm 0.15$ & $1.13 \pm 0.01$ & 23 & $4 \pm$ & 7.7 & 04 & \\
\hline 029 & .73 & & 0.078 & 1392 & & $1.12 \pm 0.05$ & & $1 \pm 0.07$ & 72 & 10.00 & $\checkmark$ \\
\hline A2065 & 230.61 & 27.70 & 0.072 & 1160 & $5.36 \pm 0.20$ & $0.60 \pm 0.02$ & 15 & $0.39 \pm 0.05$ & $4.78 \pm 0.28$ & 0.02 & $\checkmark$ \\
\hline 163 & 5 & -6.13 & 0.203 & 1781 & $13.40 \pm 0.45$ & $3.17 \pm 0.04$ & 42.51 & 21 & $19.68 \pm 0.48$ & .15 & \\
\hline 204 & 8 & & 0.152 & 1345 & 21 & $1.09 \pm$ & & $1 \pm$ & 15 & 15 & $\checkmark$ \\
\hline 18 & 9 & 66.21 & 0.171 & 1151 & $3 \pm$ & $0.73 \pm 0$ & 10 & 66 & 08 & 03 & \\
\hline A2219 & 0.10 & 46.71 & 0.228 & 1473 & $9.37 \pm 0.22$ & $1.74 \pm 0.02$ & 16.33 & $2.34 \pm 0.14$ & $11.44 \pm 0.20$ & 14.94 & \\
\hline A2256 & 6.13 & 78.63 & 0.058 & 1265 & $6.40 \pm 0.25$ & $0.78 \pm 0.02$ & $4.98 \pm 0.23$ & $0.71 \pm 0.04$ & $6.11 \pm 0.40$ & 3.92 & \\
\hline A2255 & 8.24 & 64.05 & 0.081 & 1169 & $5.79 \pm 0.15$ & $0.59 \pm 0.01$ & $3.42=$ & $0.52 \pm 0.04$ & $4.91 \pm 0.09$ & $2.47 \pm$ & \\
\hline RXC J1720.1+2638 & 260.03 & 26.61 & 0.164 & 1165 & $5.78 \pm 0.12$ & $0.70 \pm 0.01$ & $4.02=$ & $0.63 \pm 0.08$ & $5.28 \pm 0.08$ & $9.14=$ & $\checkmark$ \\
\hline A2261 & 50.61 & 32.14 & 0.224 & 1216 & $6.23+0.55$ & $0.93 \pm 0.04$ & $579-$ & $18+0$ & $6.41+1$ & $9.97-$ & $\checkmark$ \\
\hline A2390 & 328.41 & 17.69 & 0.231 & 1423 & $8.89 \pm 0.24$ & $1.54 \pm 0.02$ & $13.68 \pm 0.46$ & $1.66 \pm 0.13$ & $10.35 \pm 0.20$ & $17.20 \pm$ & $\checkmark$ \\
\hline A3827 & 0.46 & -59.95 & 0.099 & 1210 & $.19 \pm 0.10$ & $0.69 \pm 0.01$ & $4.28 \pm 0.09$ & $0.63 \pm 0.05$ & $5.55 \pm 0.07$ & 4.62 & \\
\hline $\mathrm{RXCJ} 22$ & 334.46 & -35.73 & 0.149 & 1034 & $4.68 \pm 0.10$ & $0.44 \pm 0.01$ & $2.05 \pm 0.05$ & $0.35 \pm 0.06$ & $3.64 \pm 0.06$ & $2.98 \pm 0.01$ & \\
\hline RXC J2218.6-3853 & 334.68 & -38.89 & 0.141 & 1147 & $6.19 \pm 0.19$ & $0.57 \pm 0.01$ & $3.51 \pm 0.13$ & $0.34 \pm 0.06$ & $4.92 \pm 0.11$ & $2.74 \pm 0.02$ & \\
\hline RXC J2228. & 337.12 & 20.62 & 0.412 & 1256 & $8.16 \pm 0.30$ & $1.33 \pm 0.02$ & $10.86 \pm 0.52$ & $1.34 \pm 0.23$ & $8.73 \pm 0.24$ & $11.96 \pm 0.10$ & \\
\hline RXC J2234.5-3744 & 338.62 & -37.75 & 0.151 & 1307 & $7.34 \pm 0.12$ & $0.99 \pm 0.01$ & $7.24 \pm 0.15$ & $0.90 \pm 0.07$ & $7.37 \pm 0.09$ & $7.21 \pm 0.05$ & \\
\hline MACS J2243.3-0935 & 340.84 & -9.58 & 0.444 & 1256 & $7.98 \pm 0.12$ & $1.47 \pm 0.01$ & $11.75 \pm 0.22$ & $1.91 \pm 0.24$ & $9.06 \pm 0.10$ & $14.05 \pm 0.05$ & \\
\hline A3911 & 341.60 & -52.72 & 0.097 & 1066 & $4.52 \pm 0.06$ & $0.48 \pm 0.01$ & $2.16 \pm 0.04$ & $0.38 \pm 0.04$ & $3.78 \pm 0.03$ & $2.45 \pm 0.01$ & \\
\hline AS1063 & 342.21 & -44.53 & 0.347 & 1456 & $10.73 \pm 0.25$ & $1.89 \pm 0.02$ & $20.33 \pm 0.58$ & $2.21 \pm 0.16$ & $12.60 \pm 0.20$ & $26.32 \pm 0.13$ & \\
\hline A3921 & 342.49 & -64.42 & 0.094 & 1071 & $5.01 \pm 0.07$ & $0.44 \pm 0.01$ & $2.20 \pm 0.02$ & $0.36 \pm 0.03$ & $3.82 \pm 0.03$ & $2.57 \pm 0.02$ & \\
\hline
\end{tabular}

Notes. The temperature $T_{\mathrm{X}}$ is measured in the [0.15-0.75] $R_{500}$ region, and the luminosity $L_{\mathrm{X} .500}$ is measured interior to $R_{500}$ in the [0.1-2.4] keV band. The mass $M_{500}$ is estimated from the $M_{500}-Y_{\mathrm{X}, 500}$ relation given in Eq. (1). The final column indicates whether the cluster is classified as a cool core system, defined as described in Sect. 3.4. 


\subsubsection{Impact of assumptions on pressure profile and scaling}

The blind SZ signal detection method used to detect and extract the ESZ clusters from the Planck survey (Planck Collaboration 2011d) implements the universal pressure profile from Arnaud et al. (2010). More specifically, the baseline model makes use of the generalised NFW profile fit to the 31 individual REXCESS cluster pressure profiles, after removal of the mass dependence by scaling according to the $M_{500}-Y_{\mathrm{X}, 500}$ relation given in Eq. (1). However, Arnaud et al. showed that the scatter of the individual cluster pressure profiles about the universal form increases toward the central regions, since cool core systems are more peaked, and morphologically disturbed systems are shallower, respectively, than the mean. In their Appendix C, Arnaud et al. give the best fitting GNFW model parameters for the average scaled profiles of the REXCESS cool core and morphologically disturbed subsamples.

As our cluster sample contains both cool core and morphologically disturbed systems, it is pertinent to investigate the effect of the baseline pressure profile assumption on the resulting $Y_{500}$ values. We thus re-ran the $Y_{500}$ extraction process separately for each object using the cool core and morphologically disturbed cluster profiles given in Appendix $\mathrm{C}$ of Arnaud et al. (2010). The X-ray size $\theta_{500}$ is kept the same in each case so that we are investigating the impact of the pressure profile shape within a fixed aperture. Figure 3 shows the ratio of the $Y_{500}$ of the cool core and morphologically disturbed profile extractions to that of the baseline model.

Clear trends are seen in both cases: the ratio tends to increase (decrease) with $\theta_{500}$ if the morphologically disturbed (cool core) profile is used instead of the baseline universal profile. Up to $\theta_{500} \sim 10^{\prime}$ the ratio differs from unity only by 2 per cent on average. Beyond $\theta_{500} \sim 10^{\prime}$, the derived $Y_{500}$ starts to differ gradually from the baseline value. This effect can be traced to the influence of the Planck angular resolution. Since the SZ signal extraction uses all Planck-HFI channels, the effective angular resolution is that of the channel with the largest FWHM ( $\sim 10$ arcmin at $100 \mathrm{GHz}$ ). Below this angular scale the profile shape is washed out by the convolution with the Planck beam, while above it, clusters are increasingly well-resolved. The two panels of Fig. 3 show that at the largest $\theta_{500}$ the maximum excursion is $\sim 10$ per cent. Beyond 10 arcmin, the average excursions are $\sim 6$ and $\sim 7$ per cent, respectively, for cool-core and morphologically disturbed profiles. Note that the effect is symmetric, in that for large $\theta_{500}$ a cool core profile and a morphologically disturbed profile return a value of $Y_{500}$ that differs from the baseline value by approximately the same amount, but the former is lower and the latter is higher.

In the following, the difference in $Y_{500}$ derived from extraction with the cool core and morphologically disturbed cluster profiles is added in quadrature to the uncertainty on the $Y_{500}$ from the baseline extraction. We expect this conservative error estimate to account for any difference in the underlying pressure profile shape from the universal baseline model. As detailed below in Sect. 5, we have further checked the effect of the pressure profile assumption on the derived scaling relation fits, finding it to be entirely negligible.

\section{SZ scaling relations}

We fitted the parameters governing a scaling relation between $D_{\mathrm{A}}^{2} Y_{500}$, the spherically-integrated SZ signal within $R_{500}$, and its $\mathrm{X}$-ray analogue $Y_{\mathrm{X}, 500}$. We also fitted parameters governing scaling relations between $D_{\mathrm{A}}^{2} Y_{500}$ and various other $\mathrm{X}$-ray-derived quantities including $M_{\mathrm{g}, 500}, T_{\mathrm{X}}$ and $L_{\mathrm{X}, 500}$. We further investigated the relation between $D_{\mathrm{A}}^{2} Y_{500}$ and the total mass, $M_{500}$, using the $M_{500}-Y_{\mathrm{X}, 500}$ calibration given in Eq. (1).

\subsection{Fitting method}

For each set of observables $(B, A)$, we fitted a power law relation of the form $E(z)^{\gamma} D_{\mathrm{A}}^{2} Y_{500}=10^{A}\left[E(z)^{K} X / X_{0}\right]^{B}$, where $E(z)$ is the Hubble constant normalised to its present day value and $\gamma$ and $\kappa$ were fixed to their expected self-similar scalings with $z$. The fit was undertaken using linear regression in the log-log plane, taking the uncertainties in both variables into account, and the scatter was computed as described in Pratt et al. (2009). In brief, assuming a relation of the form $Y=a X+b$, and a sample of $N$ data points $\left(Y_{\mathrm{i}}, X_{\mathrm{i}}\right)$ with errors $\sigma_{Y_{\mathrm{i}}}$ and $\sigma_{X_{\mathrm{i}}}$, the raw scatter was estimated using the error-weighted distances to the regression line:

$\sigma_{\text {raw }}^{2}=\frac{1}{N-2} \sum_{i=1}^{N} w_{\mathrm{i}}\left(Y_{\mathrm{i}}-a X_{\mathrm{i}}-b\right)^{2}$

where

$w_{\mathrm{i}}=\frac{1 / \sigma_{\mathrm{i}}^{2}}{(1 / N) \sum_{i=1}^{N} 1 / \sigma_{\mathrm{i}}^{2}} \quad$ and $\quad \sigma_{\mathrm{i}}^{2}=\sigma_{Y_{\mathrm{i}}}^{2}+a^{2} \sigma_{X_{\mathrm{i}}}^{2}$.

The intrinsic scatter $\sigma_{\mathrm{i}}$ was computed from the quadratic difference between the raw scatter and that expected from the statistical uncertainties.

We use the BCES regression method (Akritas \& Bershady 1996), which takes into account measurement errors in both coordinates and intrinsic scatter in the data and is widely used in astronomical regression, giving results that may easily be compared with other data sets fitted using the same method. We fitted all relations using orthogonal BCES regression.

\subsection{Effect of point sources and choice of baseline pressure profile}

We fitted the SZ scaling relations excluding the two objects with significant radio source contamination (Sect. 4.2.1). For all relations the change in normalisation, slope and intrinsic scatter is negligible compared to their associated uncertainties. We therefore consider the contamination by radio sources to have a negligible effect on scaling relation fits and proceed with the full sample of 62 clusters.

We have also checked whether the best fitting scaling relations are affected by the choice of baseline pressure profile, as follows. For the cool core subsample, we assigned the $Y_{500}$ derived from extraction using the cool core pressure profile. Of the remaining 40 systems, for the 20 objects with the lowest central density (Fig. 2), we assigned the $Y_{500}$ value derived from extraction using the morphologically disturbed profile. We then re-fitted all the scaling relations. The resulting best fits are in full agreement with those derived from the baseline universal profile; i.e., the difference in best fitting parameters ( 2 per cent maximum) is again smaller than their respective uncertainties.

\subsection{Correction for selection bias}

It is well known that scaling-relation determinations of the sort we are considering can be biased by selection effects of Malmquist and Eddington type when a significant part of the 
Table 2. Best fitting orthogonal BCES parameters for scaling relations.

\begin{tabular}{|c|c|c|c|c|c|c|c|c|}
\hline Relation & $A_{\mathrm{obs}}$ & $B_{\mathrm{obs}}$ & $\sigma_{\log , \mathrm{i}}$ & $A_{\text {corr }}$ & $B_{\text {corr }}$ & $\gamma$ & $\kappa$ & $X_{0}$ \\
\hline$\overline{D_{\mathrm{A}}^{2} Y_{500}-c^{*} Y_{\mathrm{X}, 500}}$ & $-4.02 \pm 0.01$ & $0.95 \pm 0.04$ & $0.10 \pm 0.01$ & -4.02 & 0.96 & - & - & $1 \times 10^{-4} \mathrm{Mpc}^{2}$ \\
\hline$D_{\mathrm{A}}^{2} Y_{500}-M_{500}$ & $-4.19 \pm 0.01$ & $1.74 \pm 0.08$ & $0.10 \pm 0.01$ & -4.22 & 1.74 & $-2 / 3$ & - & $6 \times 10^{14} M_{\odot}$ \\
\hline$D_{\mathrm{A}}^{2} Y_{500}-T_{\mathrm{X}}$ & $-4.27 \pm 0.02$ & $2.82 \pm 0.18$ & $0.14 \pm 0.02$ & -4.22 & 2.92 & -1 & - & $6 \mathrm{keV}$ \\
\hline$D_{\mathrm{A}}^{2} Y_{500}-M_{\mathrm{g}, 500}$ & $-4.05 \pm 0.01$ & $1.39 \pm 0.06$ & $0.08 \pm 0.01$ & -4.03 & 1.48 & $-2 / 3$ & - & $1 \times 10^{14} M_{\odot}$ \\
\hline$D_{\mathrm{A}}^{2} Y_{500}-L[0.1-2.4]_{\mathrm{X}, 500}$ & $-4.00 \pm 0.02$ & $1.02 \pm 0.07$ & $0.14 \pm 0.02$ & -3.97 & 1.12 & $-2 / 3$ & $-7 / 3$ & $7 \times 10^{44} \mathrm{erg} \mathrm{s}^{-1}$ \\
\hline$D_{\mathrm{A}}^{2} Y_{500}-L[0.5-2]_{\mathrm{X}, 500}$ & $-3.79 \pm 0.03$ & $1.02 \pm 0.07$ & $0.14 \pm 0.02$ & -3.75 & 1.12 & $-2 / 3$ & $-7 / 3$ & $7 \times 10^{44} \mathrm{erg} \mathrm{s}^{-1}$ \\
\hline
\end{tabular}

Notes. $c^{*}=\left(\sigma_{T} / m_{e} c^{2}\right) /\left(\mu_{e} m_{p}\right)$. Relations are expressed as $E(z)^{\gamma}\left[D_{\mathrm{A}}^{2} Y_{500}\right]=10^{A}\left[E(z)^{k} X / X_{0}\right]^{B}$. The logarithmic intrinsic scatter of the relation is denoted by $\sigma_{\log , i}$.

sample lies near a selection cut (for discussions in a cluster context see e.g., Vikhlinin et al. 2009; Pratt et al. 2009; Mantz et al. 2010 and Andersson et al. 2011). We estimated the effect of the Planck SZ selection as follows. In order to impose a selection cut on the mock catalogues, we used the observed relation between $D_{\mathrm{A}}^{2} Y_{500}$ and $\mathrm{S} / \mathrm{N}$ from the region significantly above the selection cut and extrapolated below it, along with an estimate of scatter again from observations, carried out in several redshift bins. We then constructed large mock catalogues of clusters through drawing of Poisson samples from a suitably-normalised Jenkins et al. (2001) mass function; to each cluster we assigned a value of $D_{\mathrm{A}}^{2} Y_{500}$ by adopting scaling relations with scatter that are consistent with the observed values. This procedure leads to a predicted $\mathrm{S} / \mathrm{N}$ value that can be used to impose selection cuts on the mock sample. We applied it to the full 158 cluster sample as the only X-ray information needed was the position for SZ signal re-extraction.

The effect on scaling relations is then assessed by assigning further physical properties to the mock catalogue. Following the methods of the X-ray analysis, $Y_{\mathrm{X}, 500}$ is obtained directly from the mass using Eq. (1), while $M_{\mathrm{g}, 500}$ and $L_{\mathrm{X}, 500}$ are obtained from assumed input scaling relations including scatter. Finally $T_{\mathrm{X}}$ is simply obtained from $Y_{\mathrm{X}, 500} / M_{\mathrm{g}, 500}$ on a cluster-by-cluster basis. The input scaling relation slopes and amplitudes are then adjusted until the mock observed samples match those recovered from actual data in Table 2. The input slopes then provide an estimate of the bias-corrected slope that would have been obtained had the bias been absent. The original and bias-corrected estimates are shown in Figs. 4 and 5, and the best-fitting parameters for each relation are given in Table 2. Note that the slopes of the $Y_{\mathrm{X}, 500}$ and $T_{\mathrm{X}}$ relations are derived quantities fixed by the other scalings we have chosen.

As seen in Table 2 and in Fig. 5, the importance of the Malmquist correction depends on the relation under consideration. In the case of $Y_{\mathrm{X}, 500}$ and $M_{500}$ it is negligible, due to the very small scatter seen in these relations. For the other relations, however, the Malmquist corrections can be comparable to the quoted observational uncertainties, indicating that despite the dynamic range of the Planck sample, there are biases introduced by the selection cut. The bias-corrected slopes in Table 2 are thus our best current estimates of the true underlying slopes. One should also bear in mind that the bias correction itself carries uncertainty, which we have not been able to estimate, and this increases the uncertainty on the underlying slope.

Note in particular that the bias correction leaves the $Y_{500}-Y_{\mathrm{X}, 500}$ relation completely consistent with the expected slope of unity, while the relation to $M_{500}$ remains consistent with a slope $5 / 3$.

\section{Discussion}

\section{1. $S Z$ flux vs. $X$-ray prediction}

Figure 4 shows the fundamental relation probed by the present study, that between the measured quantities $Y_{\mathrm{X}, 500}$ and $Y_{500}$. We recall that the link between these two quantities is sensitive to the structure in temperature and density. Note that X-ray information is used to determine the radius of integration for the SZ signal (i.e., $R_{500}$ ) and its overall shape (i.e., the underlying universal pressure profile). However, as we have shown above in Sect. 4.2.2, the amplitude of the SZ signal is relatively insensitive to the assumed pressure profile shape, so that the use of $\mathrm{X}$-ray priors reduces to a choice of integration aperture. Thus we regard the X-ray and SZ quantities as quasi-independent.

In the left-hand panel of Fig. 4 the relation is plotted in units of $\operatorname{arcmin}^{2}$, and shows the excellent agreement between the observed $Y_{500}, Y_{\mathrm{X}, 500}$ and the X-ray prediction from REXCESS (dashed line). Indeed, fitting the relation with the slope fixed to unity yields a normalisation $Y_{500} / Y_{\mathrm{X}, 500}=0.95 \pm 0.03$, perfectly consistent with the value $0.924 \pm 0.004$ found for the REXCESS sample (Arnaud et al. 2010), and less than unity as expected for radially-decreasing temperature profiles (e.g., Pratt et al. 2007). Furthermore, the relation is quite tight (see below), and there is no indication that cool core systems differ systematically from the other systems.

The right-hand panel of Fig. 4 shows the relation between $Y_{\mathrm{X}, 500}$ and the spherically-integrated Compton parameter $D_{\mathrm{A}}^{2} Y_{500}$. Note in particular that the slope of the bias-corrected relation is completely consistent with unity, and that the intrinsic scatter (calculated as described above in Eqs. (2) and (3)) is exceptionally small, $\sigma_{\log , i}=0.09 \pm 0.01$. A comparison with recent results obtained by SPT shows a slight difference in normalisation, although it is not significant given the larger uncertainties in the latter measurement (Andersson et al. 2011).

\subsection{Scaling relations}

In this Section we investigate other relations between $D_{\mathrm{A}}^{2} Y_{500}$ and X-ray quantities. Note that since $M_{500}$ is derived from $Y_{\mathrm{X}, 500}$, its dependence on $D_{\mathrm{A}}^{2} Y_{500}$ is directly linked to the $D_{\mathrm{A}}^{2} Y_{500}-Y_{\mathrm{X}, 500}$ relation with the exception of differing $E(z)$ dependencies. Moreover, $M_{\mathrm{g}, 500}$ and $T_{\mathrm{X}}$ are not independent; they are related via Eq. (1). It is still useful to investigate these relations, though, both for completeness and for comparison to recent results from ground-based studies.

Relations between $D_{\mathrm{A}}^{2} Y_{500}$ and gas mass $M_{\mathrm{g}, 500}$ and the X-ray temperature $T_{\mathrm{X}}$ are shown in the upper panels of Fig. 5. The bias-corrected relations yield slopes that are consistent with self-similar (5/3 and 5/2, respectively) to high accuracy. Scatter about the $D_{\mathrm{A}}^{2} Y_{500}-M_{\mathrm{g}, 500}$ relation is small, at 

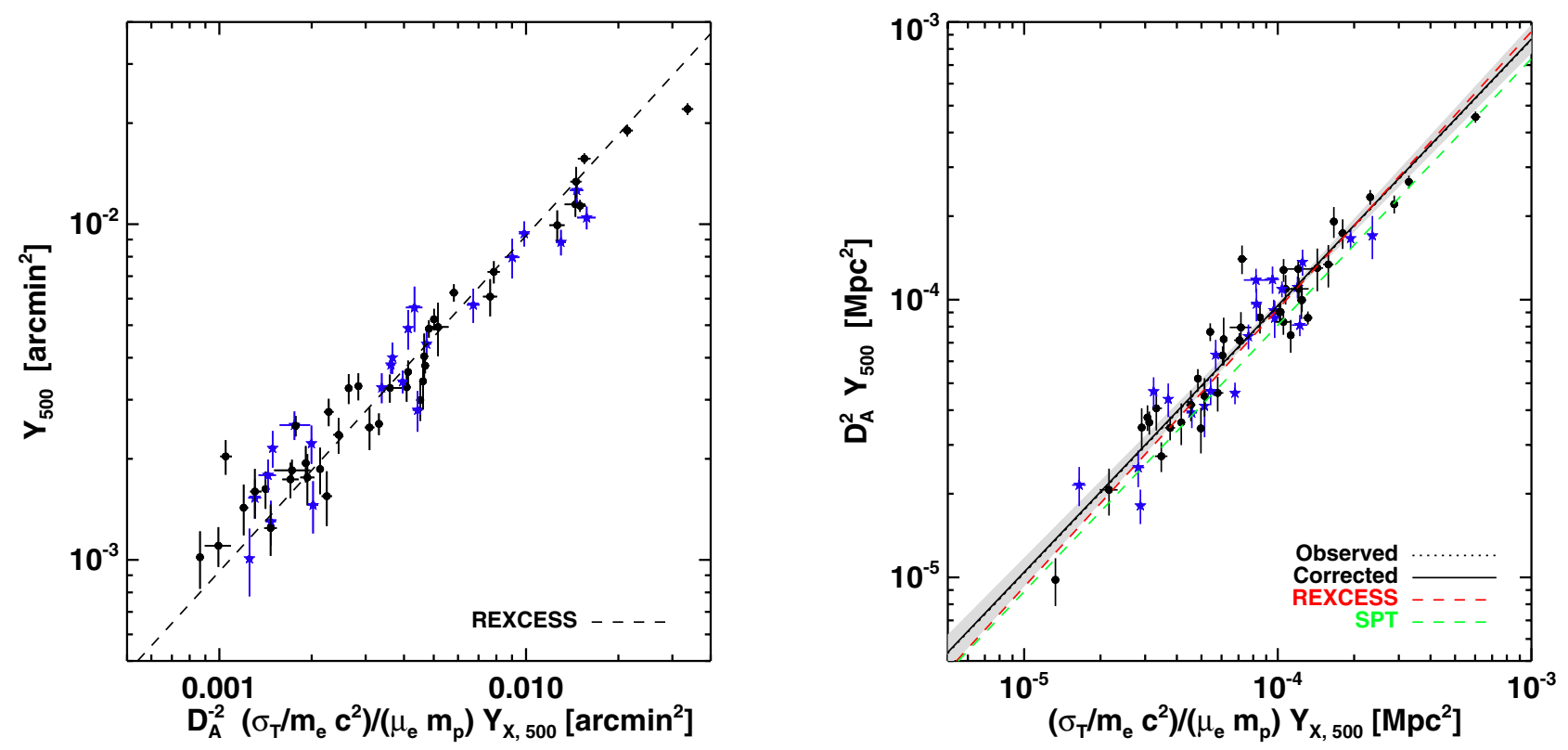

Fig. 4. SZ flux vs. X-ray prediction. Blue stars indicate cool core systems. Left panel: relation plotted in units of arcmin ${ }^{2}$. The dashed line is the prediction from REXCESS X-ray observations (Arnaud et al. 2010). Right panel: relation plotted in units of Mpc ${ }^{2}$. The SPT results are taken from Andersson et al. (2011).

$\sigma_{\log , \mathrm{i}}=0.08 \pm 0.01$, while that about the $D_{\mathrm{A}}^{2} Y_{500}-T_{\mathrm{X}}$ relation is among the largest of the relations, at $\sigma_{\text {log,i }}=0.14 \pm 0.02$. Once again, cool core systems are fully consistent with the other clusters, and there is no particular evidence that cool core systems show less scatter than the sample as a whole.

The bottom left-hand panel of Fig. 5 shows the relation between $D_{\mathrm{A}}^{2} Y_{500}$ and mass. Here again, the slope of the biascorrected relation is fully consistent with self-similar (5/3). The scatter is small $\left(\sigma_{\log , \mathrm{i}}=0.10 \pm 0.01\right)$, although it is a lower limit since the scatter between $Y_{\mathrm{X}, 500}$ and total mass is not taken into account in deriving $M_{500}$ (it is in fact the same as that about the $D_{\mathrm{A}}^{2} Y_{500}-Y_{\mathrm{X}, 500}$ relation except for the different $E(z)$ scaling). Both slope and normalisation are in excellent agreement with X-ray predictions from REXCESS, as expected from the good agreement in the $D_{\mathrm{A}}^{2} Y_{500}-Y_{\mathrm{X}, 500}$ relation. The slight offset in normalisation of the relation found by SPT (Andersson et al. 2011) can be explained by the different calibration of the $M_{500}-Y_{\mathrm{X}, 500}$ relation used in their study; it is not a significant offset given their larger normalistion uncertainties.

The ease of detecting clusters through their X-ray emission makes the X-ray luminosity an important quantity, and its calibration with the SZ signal is imperative for maximising the synergy between the Planck all-sky survey and previous all-sky $\mathrm{X}$-ray surveys such as the RASS and the upcoming eROSITA survey. The slope of the $D_{\mathrm{A}}^{2} Y_{500}-L_{\mathrm{X}, 500}$ relation for the present sample, $1.12 \pm 0.08$, is in excellent agreement with the slope predicted from X-ray observations alone $(1.07 \pm 0.08$, Arnaud et al. 2010 from REXCESS), and the normalisation is also consistent within the uncertainties. The slight offset in the best fitting normalisation for the present sample relative to the REXCESS prediction can be attributed to the relative lack of strong cooling core clusters in the present sample compared to REXCESS (see Fig. 2). The scatter, $\sigma_{\log , i}=0.14 \pm 0.02$, is largest about this relation due to the influence of cool cores, which are segregated from the other systems and all lie to the high-luminosity side. Indeed, as Fig. 5 shows and Table 2 quantifies, while the vast majority of the dispersion about the $D_{\mathrm{A}}^{2} Y_{500}-L_{\mathrm{X}, 500}$ relation is due to cool cores, these systems do not contribute significantly to the dispersion about the $D_{\mathrm{A}}^{2} Y_{500}-Y_{\mathrm{X}, 500}$ relation. Thus while the $\mathrm{X}$-ray luminosity is very sensitive to the presence of cool cores, $D_{\mathrm{A}}^{2} Y_{500}$ appears to be less so.

The slope of our best fitting $D_{\mathrm{A}}^{2} Y_{500}-L_{\mathrm{X}, 500}$ relation is also fully consistent within $1 \sigma$ with that derived by Planck Collaboration (2011f), which is based on a bin-averaging analysis at the position of known X-ray clusters in the MCXC (Piffaretti et al. 2011). As X-ray selection is more sensitive to the presence of cool cores (due to the density squared dependence of the X-ray luminosity), one might expect the Planck Collaboration (2011f) best-fitting $D_{\mathrm{A}}^{2} Y_{500}-L_{\mathrm{X}, 500}$ relation to be shifted to slightly higher luminosities (i.e., a slightly lower normalisation), as is seen. However, since the MCXC selection function is both complex and unknown, it is impossible to correct their relation for Malmquist bias effects. Thus some part of the normalisation difference between the two studies arises from correction for selection effects. However the good agreement between our results and those from Planck Collaboration (2011f)'s bin-averaged analysis argues that sample selection does not have a strong effect on the scaling relations derived from the latter analysis.

Finally, the results we have derived show that X-ray and SZ measurements give a fully coherent view of cluster structure out to moderately large scales. In particular, they indicate that effects of clumping in the X-ray gas are not significant, at least in the mass and radial range we have probed in the present study. Furthermore, the excellent agreement between the observed $D_{\mathrm{A}}^{2} Y_{500}-Y_{\mathrm{X}, 500}$ relation and the $\mathrm{X}$-ray predictions argue that the SZ and X-ray calibrations we have used are fundamentally sound.

\section{Conclusions}

We have presented SZ and X-ray data from a sample of 62 local $(z<0.5)$ galaxy clusters detected at high $\mathrm{S} / \mathrm{N}$ in the Planck survey and observed by XMM-Newton. The objects range over 

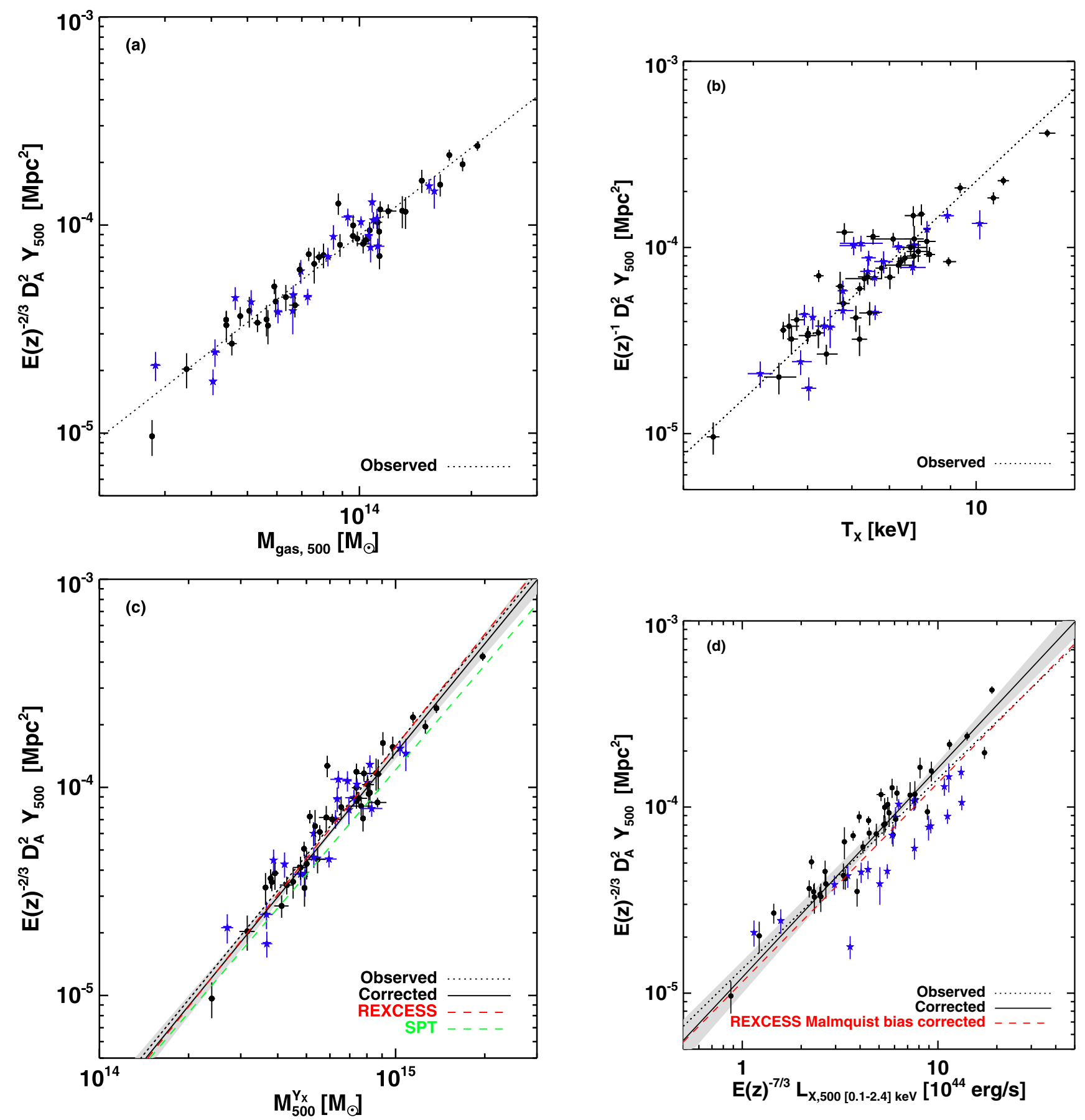

Fig. 5. Scaling relations for the 62 clusters in the Planck-XMM-Newton archive sample; fits are given in Table 2. Cool core systems are plotted as blue stars, other systems as black dots. In the upper panels, the dotted line denotes the observed scaling relation fit. In the lower panels, the dotted line denotes the observed scaling relation fit, while the solid line shows the fit once the effects of selection bias are taken into account. The grey shaded area indicates the $1 \sigma$ uncertainty. The SPT results are taken from Andersson et al. (2011).

approximately a decade in mass $\left(M_{500} \sim 2-20 \times 10^{14} M_{\odot}\right)$, and, while the sample is neither representative nor complete, it constitutes the largest, highest-quality SZ-X-ray data set currently available. This study has been undertaken in the framework of a series of papers on cluster astrophysics based on the first Planck all-sky data set (Planck Collaboration 2011d,e,f,h).

$\mathrm{SZ}$ and X-ray quantities have been extracted within $R_{500}$ and we have presented a detailed study of the resulting SZ scaling relations. Moreover, we have investigated how selection effects influence the results of the scaling relation fits. Their influence is subtle, but the slopes and normalisations of the scaling relations are generally in good agreement with X-ray predictions and other results after accounting for the selection effects. For the fundamental $D_{\mathrm{A}}^{2} Y_{500}-Y_{\mathrm{X}, 500}$ relation, we measure a remarkably small logarithmic intrinsic scatter of only $(10 \pm 1)$ per cent, consistent with the idea that both quantities are low-scatter mass proxies.

The results are fully consistent with the predictions from X-ray observations (Arnaud et al. 2010) and with recent measurements from a smaller sample spanning a wider redshift range observed with SPT (Andersson et al. 2011). The results are also in excellent agreement with the statistical analysis undertaken 

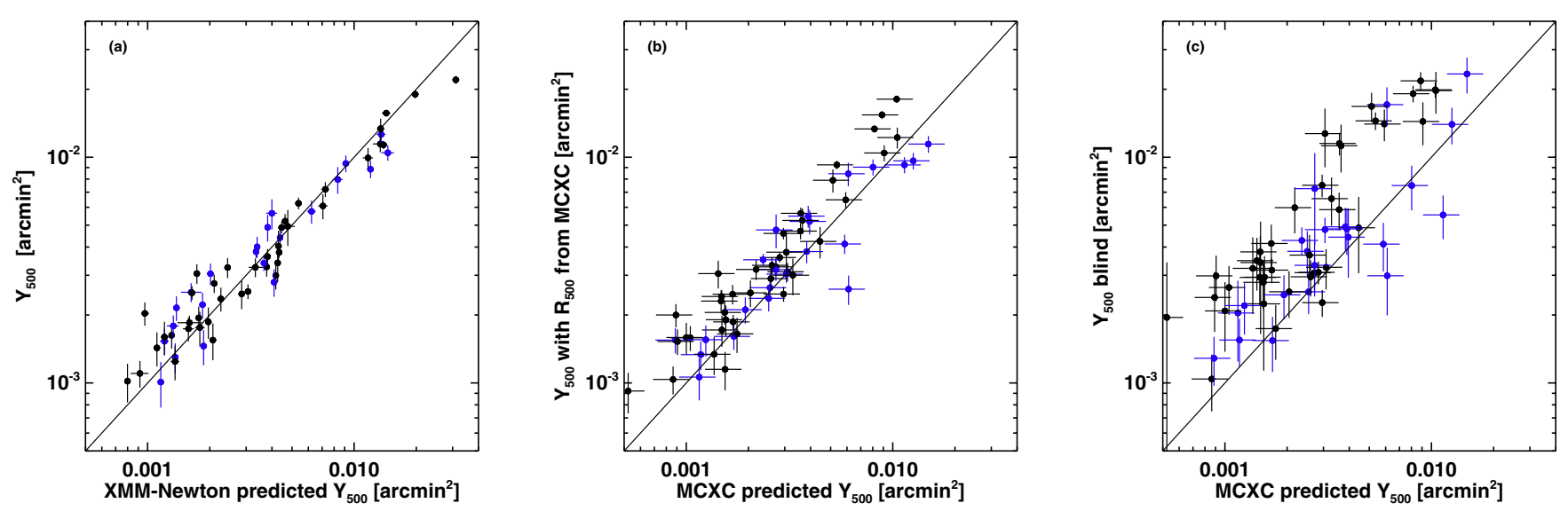

Fig. 6. Comparison of the Planck measured SZ flux, $Y_{500}$ and the predictions from X-ray measurements, $\left(\sigma_{T} / m_{\mathrm{e}} c^{2}\right) /\left(\mu_{\mathrm{e}} m_{\mathrm{p}}\right) D_{\mathrm{A}}^{-2} Y_{\mathrm{X}, 500}$. Cool core systems are marked as blue stars, other systems as black dots. (Left) $Y_{\mathrm{X}, 500}$ from XMM-Newton (see top-left panel of Fig. 5), and $Y_{500}$ computed at the position and $\theta_{500}$ derived from XMM-Newton measurements. (Middle) $Y_{\mathrm{X}, 500}$ and $Y_{500}$ respectively from $L_{\mathrm{X}, 500}$, and position and $\theta_{500}$ as given in the MCXC (Piffaretti et al. 2011). (Right) $Y_{\mathrm{X}, 500}$ from $L_{\mathrm{X}, 500}$ as given in the MCXC, while $Y_{500}$ are blind Planck measurements.

at the positions of known X-ray clusters (Planck Collaboration 2011f). This excellent agreement between observed SZ quantities and X-ray-based predictions underlines the robustness and consistency of our overall view of ICM properties. It is difficult to reconcile with the claim, based on a recent WMAP 7-year analysis, that X-ray data over-predict the SZ signal (Komatsu et al. 2011).

The results presented here, derived from only 62 systems, provide a maximally-robust local reference for evolution studies or for the use of SZ clusters for cosmology. Overall, the agreement between the present results, ground-based results and $\mathrm{X}$-ray predictions augurs well for our understanding of cluster astrophysics and for the use of clusters for cosmology. Future work will make use of the individual pressure profile shape as derived from X-rays to further improve the SZ flux extraction. Comparison of X-ray and SZ pressure profiles will also be undertaken, as will comparison of measurements with independent mass estimation methods. We will also extend our analysis to the full Planck catalogue, observing higher-redshift systems, to constrain evolution, and lower-mass objects, to better probe cluster astrophysics.

Acknowledgements. The Planck Collaboration acknowledges the support of: ESA; CNES and CNRS/INSU-IN2P3-INP (France); ASI, CNR, and INAF (Italy); NASA and DoE (USA); STFC and UKSA (UK); CSIC, MICINN and JA (Spain); Tekes, AoF and CSC (Finland); DLR and MPG (Germany); CSA (Canada); DTU Space (Denmark); SER/SSO (Switzerland); RCN (Norway); SFI (Ireland); FCT/MCTES (Portugal); and DEISA (EU). The present work is partly based on observations obtained with XMM-Newton, an ESA science mission with instruments and contributions directly funded by ESA Member States and the USA (NASA). This research has made use of the following databases: SIMBAD, operated at CDS, Strasbourg, France; the NED database, which is operated by the Jet Propulsion Laboratory, California Institute of Technology, under contract with the National Aeronautics and Space Administration; BAX, which is operated by the Laboratoire d'Astrophysique de Tarbes-Toulouse (LATT), under contract with the Centre National d'Etudes Spatiales (CNES). A description of the Planck Collaboration and a list of its members, including the technical or scientific activities in which they have been involved, can be found at http://www.rssd.esa.int/Planck.

\section{Appendix A: Optimised SZ extraction and comparison with $\mathrm{X}$-ray predictions}

As discussed in the main text, with the present cluster sample we have optimised the SZ photometry by using the higher-quality estimate of the X-ray size $\theta_{500}$, derived from $R_{500}$ measured using the $M_{500}-Y_{\mathrm{X}, 500}$ relation as detailed in Sect. 3.3.

In Fig. 6 we examine the change in $Y_{500}$ when derived using different characteristic sizes $\theta_{500}$ to extract the SZ signal. We also compare the $\mathrm{SZ}$ signal predicted using $X$-ray observations (from the $Y_{500} / Y_{X, 500}$ relation of Arnaud et al. 2010) to the observed SZ signal. In all cases, the ICM pressure is assumed to follow the baseline universal profile of Arnaud et al. (2010). As extensively described in Planck Collaboration (2011d), the SZ flux $Y_{500}$ is computed by integrating along the line-of-sight and normalising the universal pressure profile. Each profile is truncated at $5 \times R_{500}$, effectively giving a measure of the flux within a cylinder of aperture radius $5 \times R_{500}$, and then converted to the value in a sphere of radius $R_{500}$ for direct comparison with the $\mathrm{X}$-ray prediction.

The left hand panel (a) of Fig. 6 shows the relation between $Y_{500}$ and that predicted from XMM-Newton observations as used in the present paper, illustrating the tight agreement between the two quantities. We recall that here, the $R_{500}$ within which the SZ signal is extracted is derived from the measured $Y_{\mathrm{X}, 500}$ using the $M_{500}-Y_{\mathrm{X}, 500}$ relation given in Eq. (1).

The middle panel (b) of Fig. 6 assumes that only the X-ray position and luminosity of the cluster are known. In this case the mass is derived from the $M_{500}-L_{\mathrm{X}, 500}$ relation as described in the MCXC of Piffaretti et al. (2011), thus yielding the characteristic size used to extract the SZ signal, $\theta_{500}$. This mass is also used to predict $Y_{\mathrm{X}, 500}$ via the $M_{500}-Y_{\mathrm{X}, 500}$ relation in Eq. (1). Consistently, the expected SZ signal is extracted from a region of size $\theta_{500}$ centred on the X-ray position given in MCXC (as in Planck Collaboration 2011f).

In the right hand panel (c) of Fig. 6 the position and size of the cluster are unknown, thus $Y_{500}$ is devired blindly together with the SZ flux. However, the predicted SZ flux is derived as above in panel (b). The agreement between measured and predicted values clearly degrades dramatically from panels (a) to (c). Comparing panels (b) and (a), there is a systematic shift to lower predicted $Y_{\mathrm{X}, 500}$ values, with a segregation now appearing between cool cores and the other systems. This can be explained by the fact that using the luminosity as a simple mass proxy leads to an underestimate of the mass for morphologically disturbed systems in view of their position with respect to the mean $L_{X, 500}-M_{500}$ relation (Pratt et al. 2009). The inverse effect 
is seen for the cool cores. In addition, there is a smaller impact on the measured $Y_{500}$ via the effect of the assumed $\theta_{500}$. However the effect is smaller: the average ratio of XMM-Newton and MCXC characteristic sizes $\theta_{500, \mathrm{MCXC}} / \theta_{500, \mathrm{XMM}}$ is $0.95 \pm 0.06$, corresponding to a change in area of $\sim 10$ per cent, which translates into a similar variation in SZ flux. This shows that the X-ray luminosity in the MCXC is a sufficiently good mass proxy for a reliable size estimate.

Finally, panel (c) of Fig. 6 illustrates the size-flux degeneracy in blind Planck measurements. When $Y_{500}$ is measured blindly, the size is on average overestimated (see also Planck Collaboration 2011d), and so the disagreement with predictions is even more apparent.

We see that as a result of the size-flux degeneracy, an accurate estimate of the characteristic size is mandatory in order to derive an accurate measure of $Y_{500}$. A similar conclusion was reached in Planck Collaboration (2011d, see their Fig. 11), where the effect was demonstrated using the full sample of 158 clusters known in X-rays (i.e., those included in the MCXC). These authors found that, in addition to a reduction in intrinsic scatter (from 43 to 34 per cent), knowledge of the cluster size dramatically reduced the offset of the measured $Y_{500}$ to that predicted from X-rays (from 84 to 14 per cent - compare panels (c) and (b) of Fig. 6 above).

However as noted in Planck Collaboration (2011d), there is still a small but systematic discrepancy. This is mostly due to the use of $L_{X, 500}$, a quantity which shows considerable scatter with mass, as a mass proxy. The superior constraints provided by the $X M M$-Newton observations on the cluster size and on $Y_{\mathrm{X}, 500}$ suppress most of this remaining systematic effect (compare panels (b) and (a)). A smaller contribution is liked to effects due to the nature of the sample selection. This illustrates that a fully coherent approach is needed when undertaking a proper comparison between SZ and X-ray predictions.

\section{References}

Afshordi, N., Lin, Y., Nagai, D., \& Sanderson, A. J. R. 2007, MNRAS, 378, 293 Aghanim, N., Hansen, S. H., \& Lagache, G. 2005, A\&A, 439, 901

Aghanim, N., da Silva, A. C., \& Nunes, N. J. 2009, A\&A, 496, 637 Akritas, M. G., \& Bershady, M. A. 1996, ApJ, 470, 706

Andersson, K., Benson, B., Ade, P., et al. 2011, ApJ, 738, 48

Arnaud, M., Pointecouteau, E., \& Pratt, G. W. 2005, A\&A, 441, 893

Arnaud, M., Pointecouteau, E., \& Pratt, G. W. 2007, A\&A, 474, L37

Arnaud, M., Pratt, G. W., Piffaretti, R., et al. 2010, A\&A, 517, A92

Bersanelli, M., Mandolesi, N., Butler, R. C., et al. 2010, A\&A, 520, A4

Bertschinger, E. 1985, ApJS, 58, 39

Bielby, R. M., \& Shanks, T. 2007, MNRAS, 382, 1196

Bock, D., Large, M. I., \& Sadler, E. M. 1999, AJ, 117, 1578

Böhringer, H., Schuecker, P., Pratt, G. W., et al. 2007, A\&A, 469, 363

Bonamente, M., Joy, M., LaRoque, S. J., et al. 2008, ApJ, 675, 106

Bourdin, H., \& Mazzotta, P. 2008, A\&A, 479, 307

Burns, J. O., Hallman, E. J., Gantner, B., Motl, P. M., \& Norman, M. L. 2008, ApJ, 675, 1125

Carlstrom, J. E., Ade, P. A. R., Aird, K. A., et al. 2011, PASP, 123, 568

Condon, J. J., Cotton, W. D., Greisen, E. W., et al. 1998, AJ, 115, 1693

Croston, J. H., Pratt, G. W., Böhringer, H., et al. 2008, A\&A, 487, 431

da Silva, A. C., Kay, S. T., Liddle, A. R., \& Thomas, P. A. 2004, MNRAS, 348, 1401

Douspis, M., Aghanim, N., \& Langer, M. 2006, A\&A, 456, 819

Evrard, A. E., Metzler, C. A., \& Navarro, J. F. 1996, ApJ, 469, 494

Fabian, A. C., Crawford, C. S., Edge, A. C., \& Mushotzky, R. F. 1994, MNRAS, 267, 779

Jenkins, A., Frenk, C. S., White, S. D. M., et al. 2001, MNRAS, 321, 372

Jones, C., \& Forman, W. 1984, ApJ, 276, 38

Kaiser, N. 1986, MNRAS, 222, 323

Komatsu, E., Kitayama, T., Suto, Y., et al. 1999, ApJ, 516, L1

Komatsu, E., Matsuo, H., Kitayama, T., et al. 2001, PASJ, 53, 57

Komatsu, E., Smith, K. M., Dunkley, J., et al. 2011, ApJS, 192, 18

Kosowsky, A. 2003, New A Rev., 47, 939

Kravtsov, A. V., Vikhlinin, A., \& Nagai, D. 2006, ApJ, 650, 128
Lamarre, J., Puget, J., Ade, P. A. R., et al. 2010, A\&A, 520, A9

Leahy, J. P., Bersanelli, M., D’Arcangelo, O., et al. 2010, A\&A, 520, A8

Leccardi, A., \& Molendi, S. 2008, A\&A, 486, 359

Lieu, R., Mittaz, J. P. D., \& Zhang, S. 2006, ApJ, 648, 176

Mandolesi, N., Bersanelli, M., Butler, R. C., et al. 2010, A\&A, 520, A3

Mantz, A., Allen, S. W., Ebeling, H., Rapetti, D., \& Drlica-Wagner, A. 2010,

MNRAS, 406, 1773

Marrone, D. P., Smith, G. P., Richard, J., et al. 2009, ApJ, 701, L114

Maughan, B. J. 2007, ApJ, 668, 772

Melin, J., Bartlett, J. G., \& Delabrouille, J. 2006, A\&A, 459, 341

Melin, J., Bartlett, J. G., Delabrouille, J., et al. 2011, A\&A, 525, A139

Mennella, A., Maino, D., Baccigalupi, C., et al. 2011, A\&A, 536, A3

Motl, P. M., Hallman, E. J., Burns, J. O., \& Norman, M. L. 2005, ApJ, 623, L63

Nagai, D. 2006, ApJ, 650, 538

Nagai, D., Kravtsov, A. V., \& Vikhlinin, A. 2007, ApJ, 668, 1

Piffaretti, R., Arnaud, M., Pratt, G. W., Pointecouteau, E., \& Melin, J. 2011,

A\&A, 534, A109

Planck Collaboration 2011a, A\&A, 536, A1

Planck Collaboration 2011b, A\&A, 536, A2

Planck Collaboration 2011c, A\&A, 536, A7

Planck Collaboration 2011d, A\&A, 536, A8

Planck Collaboration 2011e, A\&A, 536, A9

Planck Collaboration 2011f, A\&A, 536, A10

Planck Collaboration 2011g, A\&A, 536, A11

Planck Collaboration 2011h, A\&A, 536, A12

Planck Collaboration 2011i, A\&A, 536, A13

Planck Collaboration 2011j, A\&A, 536, A14

Planck Collaboration 2011k, A\&A, 536, A15

Planck Collaboration 20111, A\&A, 536, A16

Planck Collaboration 2011m, A\&A, 536, A17

Planck Collaboration 2011n, A\&A, 536, A18

Planck Collaboration 2011o, A\&A, 536, A19

Planck Collaboration 2011p, A\&A, 536, A20

Planck Collaboration 2011q, A\&A, 536, A21

Planck Collaboration 2011r, A\&A, 536, A22

Planck Collaboration 2011s, A\&A, 536, A23

Planck Collaboration 2011t, A\&A, 536, A24

Planck Collaboration 2011u, A\&A, 536, A25

Planck Collaboration 2011v, The Explanatory Supplement to the Planck Early

Release Compact Source Catalogue (ESA)

Planck Collaboration 2011w, A\&A, 536, A26

Planck HFI Core Team 2011a, A\&A, 536, A4

Planck HFI Core Team 2011b, A\&A, 536, A6

Pointecouteau, E., Giard, M., Benoit, A., et al. 1999, ApJ, 519, L115

Pointecouteau, E., Giard, M., Benoit, A., et al. 2001, ApJ, 552, 42

Pratt, G. W., Arnaud, M., Piffaretti, R., et al. 2010, A\&A, 511, A85

Pratt, G. W., Böhringer, H., Croston, J. H., et al. 2007, A\&A, 461, 71

Pratt, G. W., Croston, J. H., Arnaud, M., \& Böhringer, H. 2009, A\&A, 498, 361

Rosset, C., Tristram, M., Ponthieu, N., et al. 2010, A\&A, 520, A13

Sehgal, N., Trac, H., Acquaviva, V., et al. 2011, ApJ, 732, 44

Staniszewski, Z., Ade, P. A. R., Aird, K. A., et al. 2009, ApJ, 701, 32

Sunyaev, R. A., \& Zeldovich, Y. B. 1972, Comments on Astrophysics and Space Physics, 4, 173

Tauber, J. A., Mandolesi, N., Puget, J., et al. 2010, A\&A, 520, A1

Vanderlinde, K., Crawford, T. M., de Haan, T., et al. 2010, ApJ, 722, 1180

Vikhlinin, A., Burenin, R. A., Ebeling, H., et al. 2009, ApJ, 692, 1033

Vikhlinin, A., Kravtsov, A., Forman, W., et al. 2006, ApJ, 640, 691

Voit, G. M. 2005, Rev. Mod. Phys., 77, 207

White, M., Hernquist, L., \& Springel, V. 2002, ApJ, 579, 16

Wik, D. R., Sarazin, C. L., Ricker, P. M., \& Randall, S. W. 2008, ApJ, 680, 17 Zacchei, A., Maino, D., Baccigalupi, C., et al. 2011, A\&A, 536, A5

1 Aalto University Metsähovi Radio Observatory, Metsähovintie 114, 02540 Kylmälä, Finland

2 Agenzia Spaziale Italiana Science Data Center, c/o ESRIN, via Galileo Galilei, Frascati, Italy

3 Astroparticule et Cosmologie, CNRS (UMR7164), Université Denis Diderot Paris 7, Bâtiment Condorcet, 10 rue A. Domon et Léonie Duquet, Paris, France

4 Astrophysics Group, Cavendish Laboratory, University of Cambridge, J J Thomson Avenue, Cambridge CB3 OHE, UK

5 Atacama Large Millimeter/submillimeter Array, ALMA Santiago Central Offices, Alonso de Cordova 3107, Vitacura, Casilla 763 0355, Santiago, Chile

6 CITA, University of Toronto, 60 St. George St., Toronto, ON M5S 3H8, Canada 
7 CNRS, IRAP, 9 Av. colonel Roche, BP 44346, 31028 Toulouse Cedex 4, France

${ }^{8}$ California Institute of Technology, Pasadena, California, USA

9 Centre of Mathematics for Applications, University of Oslo, Blindern, Oslo, Norway

10 Centro de Astrofísica, Universidade do Porto, Rua das Estrelas, 4150-762 Porto, Portugal

11 DAMTP, University of Cambridge, Centre for Mathematical Sciences, Wilberforce Road, Cambridge CB3 OWA, UK

12 DSM/Irfu/SPP, CEA-Saclay, 91191 Gif-sur-Yvette Cedex, France

13 DTU Space, National Space Institute, Juliane Mariesvej 30, Copenhagen, Denmark

14 Departamento de Física, Universidad de Oviedo, Avda. Calvo Sotelo $\mathrm{s} / \mathrm{n}$, Oviedo, Spain

15 Department of Astronomy and Astrophysics, University of Toronto, 50 Saint George Street, Toronto, Ontario, Canada

16 Department of Physics \& Astronomy, University of British Columbia, 6224 Agricultural Road, Vancouver, British Columbia, Canada

17 Department of Physics and Astronomy, University of Southern California, Los Angeles, California, USA

18 Department of Physics and Astronomy, University of Sussex, Brighton BN1 9QH, UK

19 Department of Physics, Gustaf Hällströmin katu 2a, University of Helsinki, Helsinki, Finland

20 Department of Physics, Princeton University, Princeton, New Jersey, USA

21 Department of Physics, Purdue University, 525 Northwestern Avenue, West Lafayette, Indiana, USA

22 Department of Physics, University of California, Berkeley, California, USA

23 Department of Physics, University of California, One Shields Avenue, Davis, California, USA

24 Department of Physics, University of California, Santa Barbara, California, USA

25 Department of Physics, University of Illinois at Urbana-Champaign, 1110 West Green Street, Urbana, Illinois, USA

26 Dipartimento di Fisica G. Galilei, Università degli Studi di Padova, via Marzolo 8, 35131 Padova, Italy

27 Dipartimento di Fisica, Università La Sapienza, P. le A. Moro 2, Roma, Italy

28 Dipartimento di Fisica, Università degli Studi di Milano, via Celoria, 16, Milano, Italy

29 Dipartimento di Fisica, Università degli Studi di Trieste, via A. Valerio 2, Trieste, Italy

30 Dipartimento di Fisica, Università di Ferrara, via Saragat 1, 44122 Ferrara, Italy

31 Dipartimento di Fisica, Università di Roma Tor Vergata, via della Ricerca Scientifica, 1, Roma, Italy

32 Discovery Center, Niels Bohr Institute, Blegdamsvej 17, Copenhagen, Denmark

33 Dpto. Astrofísica, Universidad de La Laguna (ULL), 38206 La Laguna, Tenerife, Spain

34 European Southern Observatory, ESO Vitacura, Alonso de Cordova 3107, Vitacura, Casilla 19001, Santiago, Chile

35 European Space Agency, ESAC, Planck Science Office, Camino bajo del Castillo, s/n, Urbanización Villafranca del Castillo, Villanueva de la Cañada, Madrid, Spain

36 European Space Agency, ESTEC, Keplerlaan 1, 2201 AZ Noordwijk, The Netherlands

37 Helsinki Institute of Physics, Gustaf Hällströmin katu 2, University of Helsinki, Helsinki, Finland

38 INAF - Osservatorio Astronomico di Padova, Vicolo dell'Osservatorio 5, Padova, Italy

39 INAF - Osservatorio Astronomico di Roma, via di Frascati 33, Monte Porzio Catone, Italy

40 INAF - Osservatorio Astronomico di Trieste, via G.B. Tiepolo 11, Trieste, Italy
41 INAF/IASF Bologna, via Gobetti 101, Bologna, Italy

42 INAF/IASF Milano, via E. Bassini 15, Milano, Italy

43 INRIA, Laboratoire de Recherche en Informatique, Université Paris-Sud 11, Bâtiment 490, 91405 Orsay Cedex, France

44 IPAG: Institut de Planétologie et d'Astrophysique de Grenoble, Université Joseph Fourier, Grenoble 1 / CNRS-INSU, UMR 5274, Grenoble, 38041, France

45 Imperial College London, Astrophysics group, Blackett Laboratory, Prince Consort Road, London, SW7 2AZ, UK

46 Infrared Processing and Analysis Center, California Institute of Technology, Pasadena, CA 91125, USA

${ }^{47}$ Institut Néel, CNRS, Université Joseph Fourier Grenoble I, 25 rue des Martyrs, Grenoble, France

48 Institut d'Astrophysique Spatiale, CNRS (UMR8617) Université Paris-Sud 11, Bâtiment 121, Orsay, France

49 Institut d'Astrophysique de Paris, CNRS UMR7095, Université Pierre \& Marie Curie, 98 bis boulevard Arago, Paris, France

50 Institute of Astronomy and Astrophysics, Academia Sinica, Taipei, Taiwan

51 Institute of Astronomy, University of Cambridge, Madingley Road, Cambridge CB3 0HA, UK

52 Institute of Theoretical Astrophysics, University of Oslo, Blindern, Oslo, Norway

53 Instituto de Astrofísica de Canarias, C/Vía Láctea s/n, La Laguna, Tenerife, Spain

54 Instituto de Física de Cantabria (CSIC-Universidad de Cantabria), Avda. de los Castros s/n, Santander, Spain

55 Jet Propulsion Laboratory, California Institute of Technology, 4800 Oak Grove Drive, Pasadena, California, USA

56 Jodrell Bank Centre for Astrophysics, Alan Turing Building, School of Physics and Astronomy, The University of Manchester, Oxford Road, Manchester, M13 9PL, UK

57 Kavli Institute for Cosmology Cambridge, Madingley Road, Cambridge, CB3 0HA, UK

58 LERMA, CNRS, Observatoire de Paris, 61 Avenue de l'Observatoire, Paris, France

59 Laboratoire AIM, IRFU/Service d'Astrophysique - CEA/DSM CNRS - Université Paris Diderot, Bât. 709, CEA-Saclay, 91191 Gif-sur-Yvette Cedex, France

${ }^{60}$ Laboratoire Traitement et Communication de l'Information, CNRS (UMR 5141) and Télécom ParisTech, 46 rue Barrault, 75634 Paris Cedex 13, France

61 Laboratoire de Physique Subatomique et de Cosmologie, CNRS/IN2P3, Université Joseph Fourier Grenoble I, Institut National Polytechnique de Grenoble, 53 rue des Martyrs, 38026 Grenoble Cedex, France

62 Laboratoire de l'Accélérateur Linéaire, Université Paris-Sud 11, CNRS/IN2P3, Orsay, France

${ }^{63}$ Lawrence Berkeley National Laboratory, Berkeley, California, USA

64 Max-Planck-Institut für Astrophysik, Karl-Schwarzschild-Str. 1, 85741 Garching, Germany

65 Max-Planck-Institut für Extraterrestrische Physik, Giessenbachstraße, 85748 Garching, Germany

${ }^{66}$ MilliLab, VTT Technical Research Centre of Finland, Tietotie 3, Espoo, Finland

${ }^{67}$ National University of Ireland, Department of Experimental Physics, Maynooth, Co. Kildare, Ireland

${ }^{68}$ Niels Bohr Institute, Blegdamsvej 17, Copenhagen, Denmark

69 Observational Cosmology, Mail Stop 367-17, California Institute of Technology, Pasadena, CA 91125, USA

70 Optical Science Laboratory, University College London, Gower Street, London, UK

71 SISSA, Astrophysics Sector, via Bonomea 265, 34136 Trieste, Italy

72 SUPA, Institute for Astronomy, University of Edinburgh, Royal Observatory, Blackford Hill, Edinburgh EH9 3HJ, UK

73 School of Physics and Astronomy, Cardiff University, Queens Buildings, The Parade, Cardiff, CF24 3AA, UK 
74 Space Research Institute (IKI), Russian Academy of Sciences, Profsoyuznaya Str, 84/32, Moscow 117997, Russia

75 Space Sciences Laboratory, University of California, Berkeley, California, USA

76 Stanford University, Dept of Physics, Varian Physics Bldg, 382 via Pueblo Mall, Stanford, California, USA

77 Universität Heidelberg, Institut für Theoretische Astrophysik, Albert-Überle-Str. 2, 69120 Heidelberg, Germany
78 Université de Toulouse, UPS-OMP, IRAP, 31028 Toulouse Cedex 4, France

79 University of Granada, Departamento de Física Teórica y del Cosmos, Facultad de Ciencias, Granada, Spain

${ }^{80}$ University of Miami, Knight Physics Building, 1320 Campo Sano Dr., Coral Gables, Florida, USA

81 Warsaw University Observatory, Aleje Ujazdowskie 4, 00-478 Warszawa, Poland 